\title{
VLT Spectropolarimetry of the Type la SN 2005ke
}

\section{A step towards understanding subluminous events ${ }^{\star}$}

\author{
F. Patat ${ }^{1}$, P. Höflich ${ }^{2}$, D. Baade ${ }^{1}$, J. R. Maund ${ }^{3}$, L. Wang ${ }^{4}$, and J. C. Wheeler ${ }^{5}$ \\ ${ }^{1}$ European Organisation for Astronomical Research in the Southern Hemisphere (ESO), Karl-Schwarzschild-Str. 2 , \\ 85748 Garching b. München, Germany \\ e-mail: fpatat@eso.org \\ 2 Department of Physics, Florida State University, Tallahassee, FL 32306-4350, USA \\ 3 Queen's University Belfast, Royal Society Research Fellow, Belfast, BT7 1NN, UK \\ 4 Department of Physics, Texas A\&M University, College Station, TX 77843, USA \\ 5 Department of Astronomy and McDonald Observatory, The University of Texas at Austin, Austin, TX 78712, USA
}

Received 1 March 2012 / Accepted 20 May 2012

\section{ABSTRACT}

\begin{abstract}
Aims. In this study we try to answer the question whether or not subluminous Type Ia supernovae have additional distinctive properties when examined from the point of view of the explosion geometry.

Methods. We have performed optical spectropolarimetric observations of the Type Ia SN 2005ke at 3 epochs (days $-8,-7$, and +76 ). The explosion properties are derived by comparing the data to explosion and radiation transfer models.

Results. The supernova shows polarimetric properties that are very similar to the only other subluminous event for which spectropolarimetry is available, i.e. SN 1999by. The data present a very marked dominant axis, which is shared by both the continuum and lines such as $\mathrm{Si}$ II $\lambda 6355$, suggesting that the relatively large, global asymmetry is common to the photosphere and the line-forming region. The maximum polarization degree observed in the $\mathrm{Si}$ II $\lambda 6355$ absorption reaches $0.39 \pm 0.08 \%$. At variance with what is seen in core-normal Type Ia, SN 2005ke displays significant continuum polarization, which grows from the blue to the red and peaks at about $7000 \AA$, reaching $\sim 0.7 \%$. The properties of the polarization and flux spectra can be understood within the framework of a subluminous delayed-detonation (DD), or pulsating DD scenario, or white dwarf (WD) mergers. The difference in appearance with respect to core-normal SNe Ia is caused by low photospheric temperatures in combination with layers of unburned $\mathrm{C}$, and more massive layers of the products of explosive $\mathrm{C}$ and $\mathrm{O}$ burning. The comparatively high level of continuum polarization is explained in terms of a significant global asymmetry $(\sim 15 \%)$, which is well reproduced by an oblate ellipsoidal geometry within the general context of a DD explosion.

Conclusions. Our results suggest that SN 2005ke arose either from a single-degenerate system in which the WD is especially rapidly rotating, close to the break-up velocity, or from a double-degenerate merger. Based on the current polarization data, we cannot distinguish between these two possibilities. Possible tests are discussed.
\end{abstract}

Key words. supernovae: general - supernovae: individual: SN2005ke - white dwarfs - dust, extinction - techniques: polarimetric

\section{Introduction}

Subluminous Type Ia Supernovae (SNe Ia) are still a puzzle in our understanding of this important class of explosive events (Hillebrandt \& Niemeyer 2000). Although they contribute to "only" $15 \%$ of all Type Ia events (Li et al. 2011), understanding their progenitors and the mechanism that powers their explosions is fundamental to fully grasp the Type Ia supernova phenomenon (see, for instance, Pakmor et al. 2010).

These objects, commonly dubbed "91bg-like" after the prototypical event SN 1991bg (Filippenko et al. 1992; Leibundgut et al. 1993), deviate quite significantly from the behavior defined by core-normal events (Branch et al. 2006). Photometrically, they are about 2 mag fainter, display rapidly declining light curves with narrower peaks, lack a secondary maximum in the near-infrared (NIR) bands, have redder colors at maximum light, and obey a different relation between maximum luminosity and light curve shape (Garnavich et al. 2004). Spectroscopically,

^ Based on observations made with ESO Telescopes at the Paranal Observatory under Program IDs 076.D-0177 and 076.D-0178. they show cool spectra, characterized by intermediate-mass elements, particularly strong $\mathrm{O}_{\mathrm{I}}$ and $\mathrm{Ti}$ II absorptions, and lower expansion velocities. In addition, both iron-group elements and silicon are spread over velocities spanning a large fraction of the ejecta, implying very substantial mixing (Taubenberger et al. 2008), as opposed to what happens in core-normal objects. The estimated mass of ${ }^{56} \mathrm{Ni}$ is as low as $0.1 M_{\odot}$ (Stritzinger et al. 2006).

All these facts pose a challenge and call for a different explosion mechanism for subluminous $\mathrm{SNe}$ Ia than for core-normal $\mathrm{SNe}$ Ia. As of today, although several theoretical scenarios have been put forward (see Pakmor et al. 2011, for a recent review), we do not have a clear idea about the origin of this sub-class of Type Ia SNe. On the observational side, there are indications that these objects form a rather homogeneous and distinct class; however, there is growing evidence that transition objects, sharing properties of both subluminous and core-normal SNe, do exist (Maguire et al. 2011).

An additional channel for investigating the diversity of $\mathrm{SNe}$ is polarimetry, which enables the study of the explosion geometry (Wang \& Wheeler 2008). Well studied 91bg-like events are 


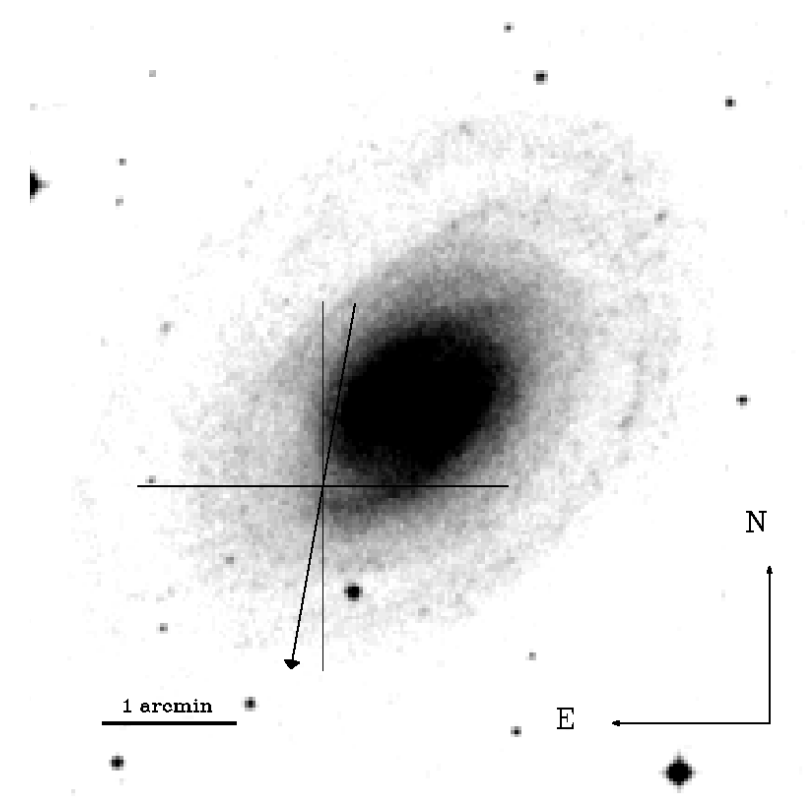

Fig. 1. A DSS image of NGC 1371. The location of SN 2005ke is marked by the cross. The arrow indicates the ISP position angle $\left(\theta_{\mathrm{ISP}}=\right.$ 170 degrees; see Sect. 4).

quite rare, both because of their lower luminosity and their intrinsic lower rate. For the same reasons, subluminous events studied through spectropolarimetry are exceptionally rare (Wang $\&$ Wheeler 2008). More precisely, so far the geometrical properties of the explosion have only been studied for one such SN, i.e. SN1999by, which showed very distinct features (Howell et al. 2001).

In this paper we present a spectropolarimetric study of another $91 \mathrm{bg}$-like event, SN 2005ke. This SN was discovered by Baek et al. (2005) on 13 November 2005 (UT) in the Virgo Cluster spiral galaxy NGC 1371 (see Fig. 1), located at a distance of $25.8 \pm 3.2 \mathrm{Mpc}$ (Wood-Vasey et al. 2008) and receding with a velocity $v_{\text {gal }}=1463 \mathrm{~km} \mathrm{~s}^{-1}$ (Koribalski et al. 2004). A few days later, the object was classified as an underluminous Type Ia event caught before maximum light (Patat et al. 2005). Spectroscopic and photometric data were presented by Immler et al. (2006), Bufano et al. (2009), Folatelli et al. (2010), and Contreras et al. (2010), to which we refer the reader for the general properties of this object.

The paper is organized as follows. In Sect. 2 we present and discuss our observations and data reduction. The spectroscopic evolution during the phases covered by our observations is analyzed in Sect. 3, while Sect. 4 presents the spectropolarimetric data. The data are compared to explosion and radiation transfer models in Sect. 5. In Sect. 6 we discuss our results, while in Sect. 7 we summarize the conclusions.

\section{Observations and data reduction}

We have observed SN 2005ke on 3 different epochs, using the FOcal Reducer/low-dispersion Spectrograph (hereafter FORS1), mounted at the Cassegrain focus of the ESO-Kueyen $8.2 \mathrm{~m}$ telescope (Appenzeller et al. 1998). In this multi-mode instrument, equipped with a $2048 \times 2048$ pixel $(p x)$ TK2048EB4-1 backside-thinned $\mathrm{CCD}$, polarimetry is performed introducing a Wollaston prism (19" throw) and a super-achromatic half-wave plate. In order to reduce some known instrumental problems (see Patat \& Romaniello 2006) we have always used 4 half-wave
Table 1. Log of VLT-FORS1 spectropolarimetric observations of SN 2005ke.

\begin{tabular}{lccccc}
\hline \hline $\begin{array}{l}\text { Date } \\
(\mathrm{UT})\end{array}$ & $\begin{array}{c}\text { MJD } \\
(\mathrm{JD}-2400000.5)\end{array}$ & $\begin{array}{c}\text { Phase } \\
(\text { days })\end{array}$ & $\begin{array}{c}V \\
(*)\end{array}$ & $\begin{array}{c}\text { Airmass } \\
(\text { average })\end{array}$ & $\begin{array}{c}\text { Exp. time } \\
(\mathrm{s})\end{array}$ \\
\hline $2005-11-16$ & 53690.10 & -8.6 & 15.2 & 1.1 & $4 \times 300$ \\
$2005-11-17$ & 53691.10 & -7.6 & 15.0 & 1.2 & $4 \times(300+600)$ \\
$2006-02-08$ & 53775.06 & +76 & 17.7 & 1.2 & $4 \times 900$ \\
\hline
\end{tabular}

Notes. (*) $V$ magnitudes are derived from Contreras et al. (2010).

plate (HWP) angles (0, 22.5, 45 and 67.5 degrees). The SN was observed at two pre-maximum phases ( -8.6 and -7.6 days) and one additional epoch (two and a half months past maximum light). Exposure times ranged from 5 to $15 \mathrm{~min}$ per plate angle and, on the second epoch, the sequence was repeated in order to increase the signal-to-noise ratio. All spectra were obtained with the low-resolution G300V grism coupled to a 1.1 arcsec slit, giving a spectral range $3300-8600 \AA$, a dispersion of $\sim 2.9 \AA$ pixel $^{-1}$ and a resolution of $12.4 \AA(F W H M)$ at $5800 \AA$. Data were bias, flat-field corrected and wavelength calibrated by means of standard tasks within IRAF ${ }^{1}$. The rms error on the wavelength calibration is about $0.7 \AA$. The wavelength scale was corrected to the rest-frame using the host galaxy recession velocity $\left(1463 \mathrm{~km} \mathrm{~s}^{-1}\right.$; Koribalski et al. 2004).

The ordinary and extraordinary beams were processed separately. Stokes parameters were computed by means of specific routines written by us, and error estimates were performed following the prescriptions described by Patat \& Romaniello (2006), while the HWP zeropoint angle chromatism was corrected using tabulated data (Jehin et al. 2002). In order to increase the signal-to-noise ratio, multiple data sets obtained at the same epoch were combined, and the final Stokes parameters binned in $\sim 52 \AA$ wide bins (20 pixels).

Flux calibration was achieved through the observation of spectrophotometric standard stars with the full polarimetric optics inserted (HWP angle set to 0 degrees). Instrumental polarization and the position angle offset were checked by observing polarized and unpolarized standard stars, obtained within the FORS1 calibration plan.

The log of observations is reported in Table 1, where the $\mathrm{SN}$ phases were computed with respect to the $B$ maximum light (JD = 2453 699.16; Folatelli et al. 2010).

\section{General properties of SN 2005ke}

From the classification spectrum it was clear that SN 2005ke was a subluminous Type Ia event (Patat et al. 2005), very similar to SN 1999by (Howell et al. 2001; Garnavich et al. 2004). The characteristic Ti II feature at about $4200 \AA$ was clearly detected in the pre-maximum spectra. The spectroscopic evolution of SN 2005ke during the epochs discussed in this paper is presented in Fig. 2. The close resemblance to another wellstudied subluminous SN Ia 2005bl (Taubenberger et al. 2008) is illustrated in Fig. 3, where for comparison we have also plotted the spectrum of the core-normal SN 1994D at the same pre-maximum epoch. The expansion velocity, deduced from the minimum of the Si II $\lambda 6355$ absorption trough on day -7 ,

\footnotetext{
1 IRAF is distributed by the National Optical Astronomy Observatories, which are operated by the Association of Universities for Research in Astronomy, under contract with the National Science Foundation.
} 


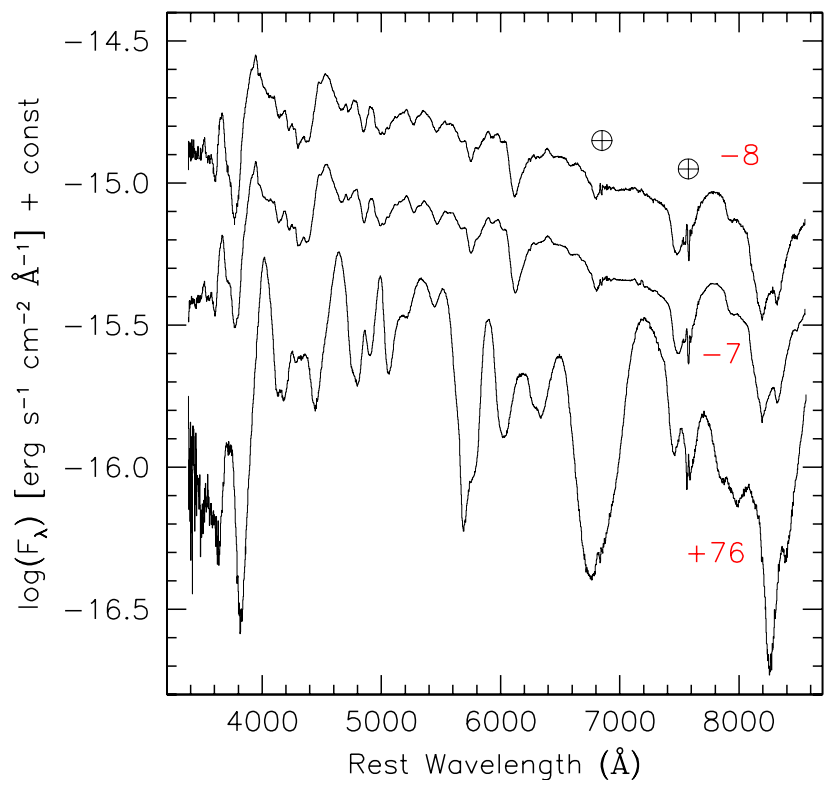

Fig. 2. Spectroscopic evolution of SN 2005ke during the epochs covered by the observations presented in this paper. Phases refer to $B$ maximum light (JD $=2453699.16$; Folatelli et al. 2010). For presentation the spectrum of the second epoch is shifted by $\log F=-0.5$.

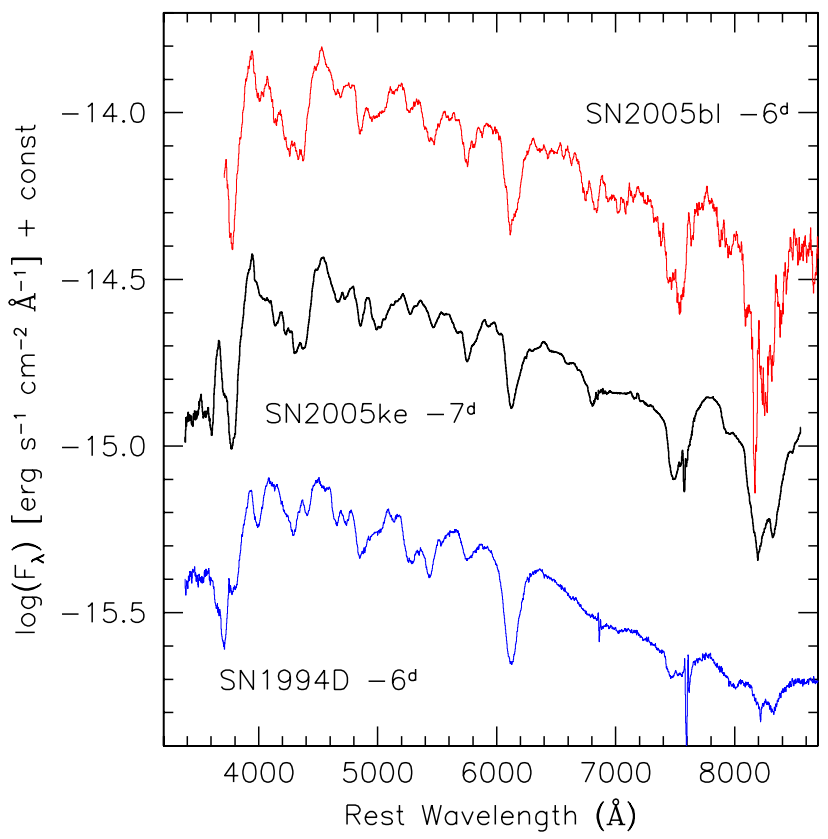

Fig. 3. Spectroscopic comparison of subluminous SNe 2005ke, 2005bl (Taubenberger et al. 2008) and core-normal SN 1994D (Patat et al. 2006) at $-7 /-6$ days.

is $11300 \mathrm{~km} \mathrm{~s}^{-1}$, very similar to the values measured for the subluminous SN 2005bl (Taubenberger et al. 2008). On the following day, the velocity dropped to about $10900 \mathrm{~km} \mathrm{~s}^{-1}$. These values are compared to those published for other subluminous Ia events in Fig. 4. Although only early measurements are available for SN 2005ke, and therefore no firm conclusion can be drawn, this object seems to conform to the behavior shown by the other $91 \mathrm{bg}$-like events. A best fit to the combined data of SN 1991 bg, 1999 by and 2005ke gives a velocity gradient $\dot{v}=$ $106 \pm 9 \mathrm{~km} \mathrm{~s}^{-1} \mathrm{day}^{-1}$.

SN 1998de displays higher velocities in the pre-maximum epochs, while a month past maximum light it falls very close to

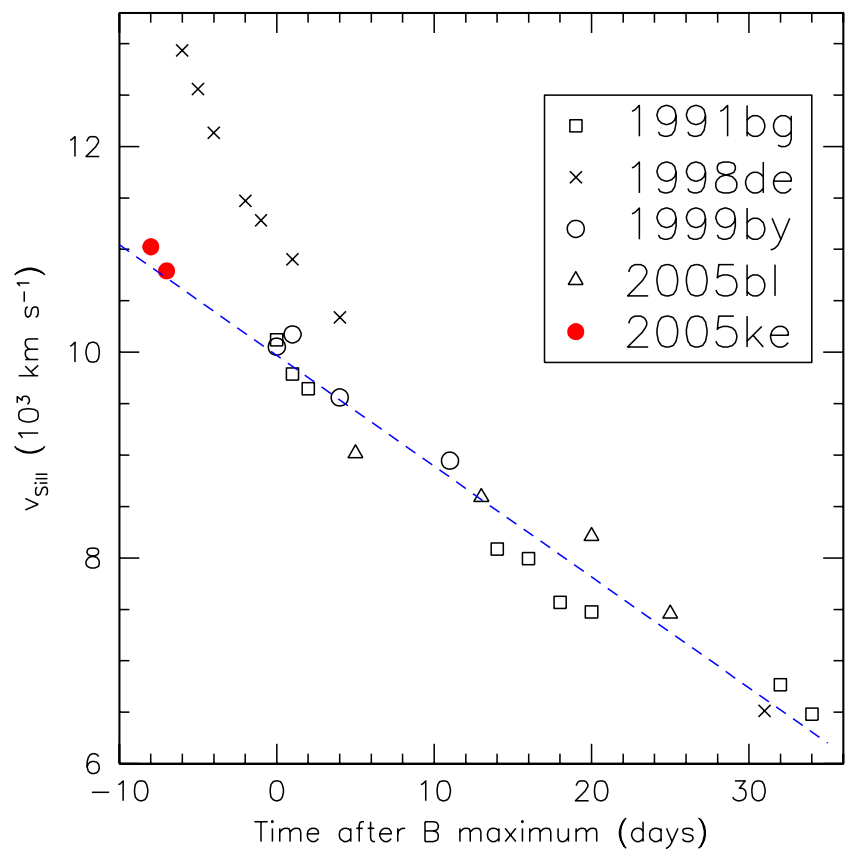

Fig. 4. Evolution of Si ir $\lambda 6355$ velocity for a compilation of underluminous Type Ia: 1991bg (Turatto et al. 1996), 1998de (Modjaz et al. 2001; Matheson et al. 2008), 1999by (Vinko et al. 2001; Howell et al. 2001), and 2005bl (Taubenberger et al. 2008). The dashed line is a best fit to the data after excluding SN1998de.

the relation derived from the previous objects. Its average velocity gradient is $\sim 175 \mathrm{~km} \mathrm{~s}^{-1}$ day $^{-1}$. Although this indicates a possible break in the homogeneity otherwise seen in this sub-class of SNe Ia (Taubenberger et al. 2008), all the objects plotted in Fig. 4 definitely qualify for the HVG class introduced by Benetti et al. (2005).

From a photometric point of view, SN 2005ke has shown the same properties as other $91 \mathrm{bg}$-like objects (see Contreras et al. 2010), i.e. the absence of a secondary peak in the red passbands, an intrinsically red color and a fast decline rate $\Delta m_{15}(B)=$ $1.76 \pm 0.01$ (Folatelli et al. 2010). For comparison, SN 1999by and SN $2005 \mathrm{bl}$ reached $\Delta m_{15}(B)=1.90 \pm 0.05$ (Garnavich et al. 2004) and $1.93 \pm 0.10$ (Taubenberger et al. 2008), respectively. SN 2005ke reached a peak magnitude of $V=14.2$ (Contreras et al. 2010), which coupled to the estimated distance modulus $\mu=32.06 \pm 0.27$ (Wood-Vasey et al. 2008) yields an absolute magnitude $M_{V}=-17.9 \pm 0.3$. We note that a fainter magnitude is obtained $\left(M_{V} \sim-17\right)$ if one uses the Tully-Fisher distance modulus ( $\mu=31.16$, Tully 1988).

\section{Spectropolarimetry}

Although we obtained two separate pre-maximum epochs (days -8.6 and -7.6), a close analysis of the data shows that there is no statistically significant evolution between the two dates. Therefore, in view of the subluminous nature of the object, we have merged the two data sets in order to maximize the signal-to-noise ratio. The result is shown in Fig. 5, where we plot the combined spectropolarimetric data set (corresponding to a total exposure time of $1200 \mathrm{~s}$ per half-wave plate angle) on the $Q-U$ plane.

The data show a dominant axis (Wang et al. 2001; Wang \& Wheeler 2008), not passing through the origin of the $Q-U$ plane, hence indicating the presence of different components, characterized by different polarization degree and position angle 


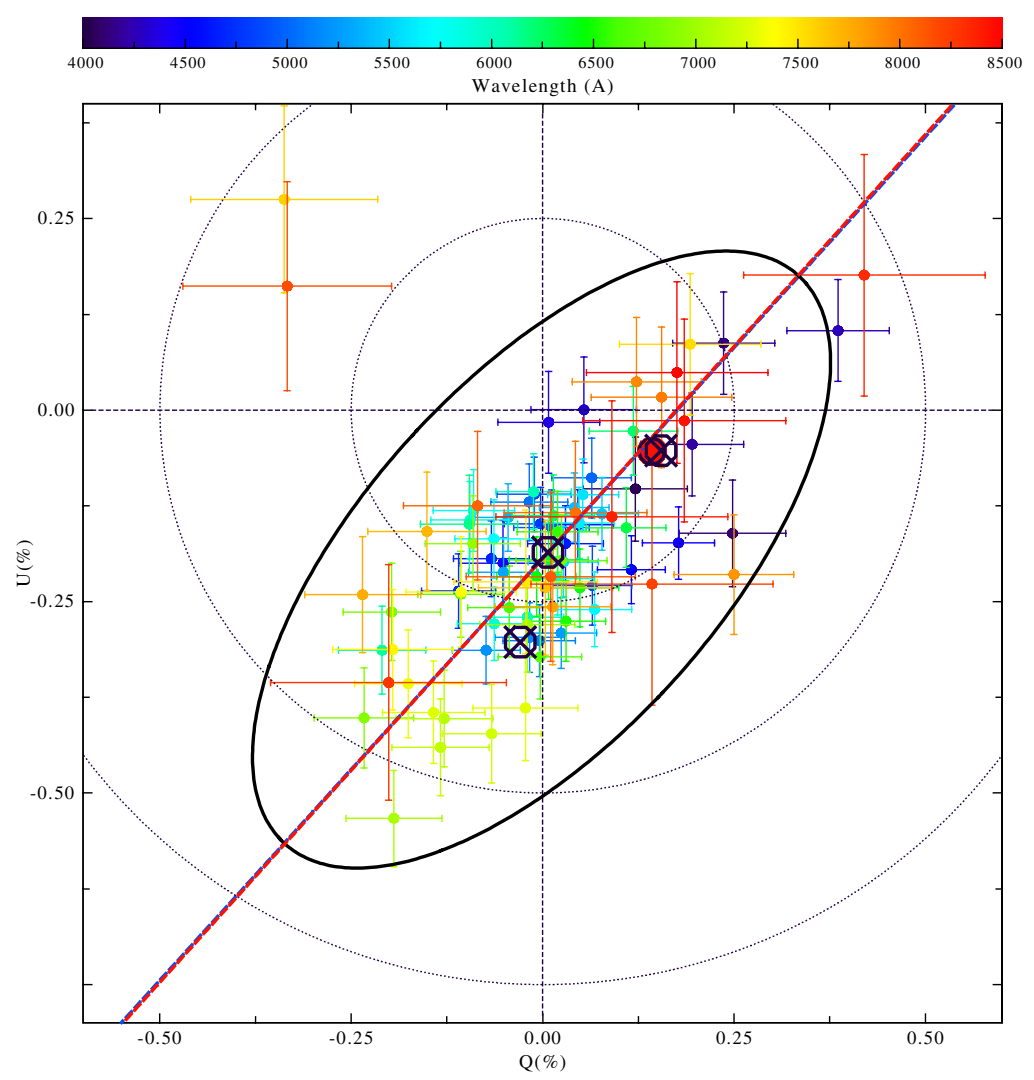

Fig. 5. Combined spectropolarimetry of SN 2005ke (November 16 and 17, 2005). The large filled circle marks the ISP estimated from the late-time data (see Sect. 4.1), while the circled crosses indicate B, A and C ISP estimates (from right to left respectively; see Table 2). The dashed line traces the best fit dominant axis, while the ellipse indicates the axial ratio and orientation of the data (see Sect. 4.2). For presentation, the size of the ellipse was arbitrarily scaled. The dotted circles indicate polarization levels of $0.25,0.50$ and $0.75 \%$.

(see for instance Patat et al. 2009b, their Appendix B). The polarization variation along the dominant direction appears to be moderate, but significant, reaching about $0.8 \%$. In contrast, the polarization variation in the perpendicular direction is consistent with the measurement errors (see Sect. 4.2).

The first step for disentangling the different contributions is the subtraction of any interstellar polarization (ISP) that might arise within aligned asymmetric dust grains along the line of sight.

\subsection{Reddening and interstellar polarization}

The Galactic reddening along the line of sight to SN 2005ke is $E_{B-V}=0.023$ (Schlegel et al. 1998). Using the relation found by Serkowski et al. (1975) this implies that the Galactic interstellar polarization (ISP) is expected to be lower than $\sim 0.2 \%$. The compilation of polarimetric data for Galactic stars by Heiles (1999) contains only one entry within 3 degrees from NGC $1371^{2}$, i.e. HD 22332. This star, at a projected distance of 1.1 degrees from SN 2005ke, has a linear polarization $P=0.13 \%$ at position angle $\mathrm{PA}=4.1$ degrees (Matheson \& Ford 1970), in good agreement with the low Galactic extinction along this line of sight. Since the extinction within the host galaxy is estimated to be comparably low $\left(E_{B-V}=0.036 \pm 0.005\right.$; Folatelli et al. 2010), the total ISP is expected to be of order of a few $0.1 \%$.

Several methods to derive the level of ISP in SN spectra have been proposed in the literature (see for instance Leonard et al. 2000; Howell et al. 2001; Wang et al. 2001), and used in

\footnotetext{
2 http://vizier.cfa.harvard.edu/viz-bin/VizieR? - source=II/226
}

a number of cases in the SN field. They are based either on the analysis of the $Q-U$ plane, or on the assumption that some portions of the SN spectra are intrinsically unpolarized (Wang et al. 1997; Howell et al. 2001; Chornock et al. 2006), so that a nonnull continuum polarization can be interpreted as arising within the interstellar medium.

Although the application of these methods is possible for this object (see below), we attempted an alternative solution, which is trying to actually measure the ISP. For this purpose we have obtained spectropolarimetry of SN 2005ke at a rather late epoch, about two and a half months past maximum light, when the SN is entering the nebular phase. Since at these epochs the continuum polarization from an asymmetric photosphere is expected to be null, any residual continuum polarization can be interpreted as arising in intervening interstellar dust. The very well-studied case of SN 2006X (Patat et al. 2009a) showed that, with only the remarkable exception of the Ca II triplet, line polarization is null at epochs later than one month past maximum light. This allows one to use a wide wavelength range in order to reduce the uncertainty.

The spectropolarimetry of SN $2005 \mathrm{ke}$ on day +76 is presented in Fig. 6. The data do not show any statistically significant polarization variation across the whole spectral range. The weighted average Stokes parameters, estimated in the wavelength range 4500-7500 $\AA$, are $\left\langle Q_{\text {ISP }}\right\rangle=0.14 \pm 0.03 \%$ and $\left\langle U_{\text {ISP }}\right\rangle=-0.05 \pm 0.03 \%$, corresponding to a polarization degree $P_{\text {ISP }}=0.15 \pm 0.03 \%$, and a position angle $\theta_{\mathrm{ISP}}=$ $170 \pm 6$ degrees. The rms deviation of single data points from the weighted average value is $0.26 \%$, i.e. fully compatible with the measurement errors. Because of the weakness of the $\mathrm{SN}$ at this 
F. Patat et al.: VLT spectropolarimetry of the Type Ia SN 2005ke

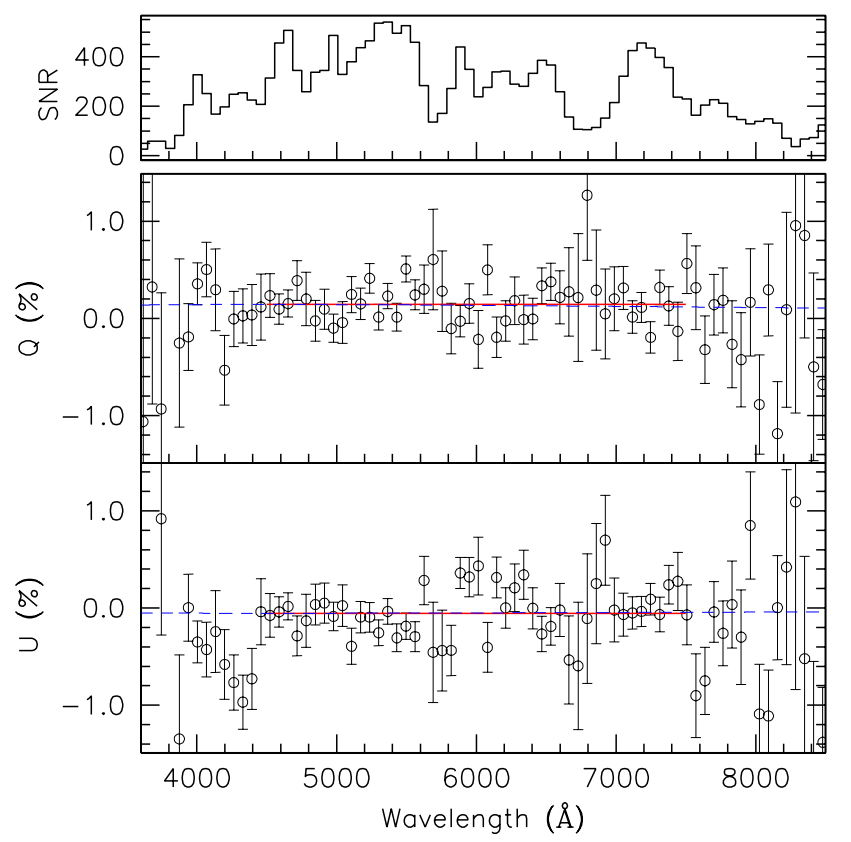

Fig. 6. Stokes parameters $Q$ (central panel) and $U$ (lower panel) on day +76 . For presentation, the data were binned to $65 \AA(25 \mathrm{px})$. The two horizontal segments mark the weighted average values within the wavelength range (4500-7500 $\AA$ ). The dashed curves trace a Serkowski-Whittet law with $P_{\max }=0.15 \%, \theta=169.7$ degrees, and $\lambda_{\max }=4500 \AA$. The upper panel presents the signal-to-noise ratio per resolution element in the intensity spectrum.

epoch $(V \sim 17.7$, i.e. almost 3 mag fainter than at maximum), it is not possible to deduce the wavelength dependency of the ISP. For a Serkowski-Whittet law (Serkowski et al. 1975; Whittet et al. 1992) the polarization variation is very mild across the optical domain, and its absolute amount is proportional to the maximum polarization. For the present case, this implies a very small $(\leq 0.05 \%)$ peak-to-peak variation across the spectral domain covered by our observations, certainly below the accuracy one can achieve with instruments like FORS (Patat \& Romaniello 2006).

In addition, we note that the ISP wavelength dependency observed in several supernovae clearly deviates from the Galactic Serkowski law (Leonard \& Filippenko 2001; Leonard et al. 2002; Maund et al. 2007a; Patat et al. 2009a,b; Maund et al. 2010a). Therefore, rather than using a Serkowski law to model the ISP, we ignored its wavelength dependency and vectorially subtracted the constant values derived above. Once placed on the $Q-U$ plane, the derived ISP happens to be very close to the dominant axis derived fitting all the data of the combined spectropolarimetry obtained in the pre-maximum epoch (Fig. 5, large filled circle). We also notice that the measured ISP is very close to one of the two possible solutions one would consider in the absence of late-time data, at either end of the data along the dominant axis (see, for instance, the ISP derivation for SN 1999by, Howell et al. 2001). More precisely, the measured ISP essentially coincides with the solution implying the lowest ISP, which is fully in line with the low reddening suffered by the SN.

As an independent check, we have derived the ISP measuring the polarization in the wavelength ranges $4800-5600 \AA$ (A), 4000-4200 $\AA$ (B), and 5100-5300 $\mathrm{A}$ (C) on the combined premaximum spectrum. These ranges were proposed by Howell et al. (2001), Chornock et al. (2006) and Maund et al. (2010a) respectively, based on the assumption that in these regions the line blanketing is sufficient to produce complete depolarization.
Table 2. ISP determinations.

\begin{tabular}{lcccc}
\hline \hline $\begin{array}{l}\text { Range } \\
(\AA)\end{array}$ & Phase & ID & $\begin{array}{c}\left\langle Q_{\text {isp }}\right\rangle \\
(\%)\end{array}$ & $\begin{array}{c}\left\langle U_{\text {isp }}\right\rangle \\
(\%)\end{array}$ \\
\hline $4800-5600$ & pre-max & A & $+0.007 \pm 0.012$ & $-0.186 \pm 0.017$ \\
$4000-4200$ & pre-max & B & $+0.155 \pm 0.040$ & $-0.053 \pm 0.047$ \\
$5100-5300$ & pre-max & C & $-0.029 \pm 0.025$ & $-0.303 \pm 0.030$ \\
\hline $4500-7500$ & late & D & $+0.143 \pm 0.029$ & $-0.054 \pm 0.029$ \\
\hline
\end{tabular}

The results are compared to those obtained from the late-time spectrum in Table 2 and Fig. 5 (circled crosses). The ISP derived from the three wavelength ranges is $\leq 0.3 \%$, which is consistent with the low level of polarization expected from the mild extinction along the line of sight to SN 2005ke. The estimate obtained for the $\mathrm{B}$ range coincides (within the statistical errors) with the late-time measurement, indicating that in this particular spectral region the depolarization assumption holds.

This is not the case (at least for SN 2005ke) for the other two wavelength intervals, which give ISP values that are inconsistent with the late-time data. In addition, the Stokes parameters derived from range A place the ISP very close to the centroid of the data set (see next section), so that notwithstanding the clear existence of a dominant axis, the implied polarization angle would become erratic (this would also turn into a very low intrinsic polarization level). As for range $\mathrm{C}$, it must be noticed that this was proposed for the peculiar SN Ia 2005hk, where this particular region was indeed expected to have null intrinsic polarization (Maund et al. 2010a). This clearly does not apply to SN 2005ke.

We will adopt the ISP derived from the late-time data throughout the paper.

Since dust grains tend to be aligned along the direction of the galactic magnetic field, which in turn follows the spiral pattern (see Scarrot et al. 1987), the ISP is expected to be tangential to the local spiral arm of the host galaxy. This has been observed, for instance, in SN 2001el (Wang et al. 2003), SN 2006X (Patat et al. 2009a), in the bright optical transient in NGC 300 (Patat et al. 2009b), and in SN 2005hk (Maund et al. 2010a). When the ISP within the host galaxy and the Milky Way are comparable, as is the case for SN 2005ke, the position angle of the resulting polarization vector can differ substantially from the direction defined by the local spiral pattern. This is the likely explanation why the derived ISP position angle is far from being parallel to the spiral arm of NGC 1371 at the location of SN 2005ke (Fig. 1). The ISP position angle ( $170 \pm 6$ degrees) is not too different from that reported for the nearby star HD 22332 (4.1 degrees; see Sect. 4.1). This indicates that the Milky Way contribution may dominate over that arising within the host.

\subsection{Intrinsic polarization of SN 2005ke}

In order to derive the fundamental parameters of the dominant axis and their statistical significance, we have performed a weighted linear squares fit to the data (accounting for errors in both $Q$ and $U$ ) in the wavelength range 4000-7500 $\AA$, using the linear relation $U=\alpha+\beta Q$. The results are presented in Table 3, where we included $\chi^{2}$ per degree of freedom (DOF), the Pearsons correlation coefficient $\left(r_{x y}\right)$, the rms deviation of data points from the best fit axis $(\sigma)$, and the dominant axis position angle $\left(\theta_{\mathrm{d}}\right)$.

In addition to the least squares analysis, we have performed a principal components analysis. This was achieved constructing 


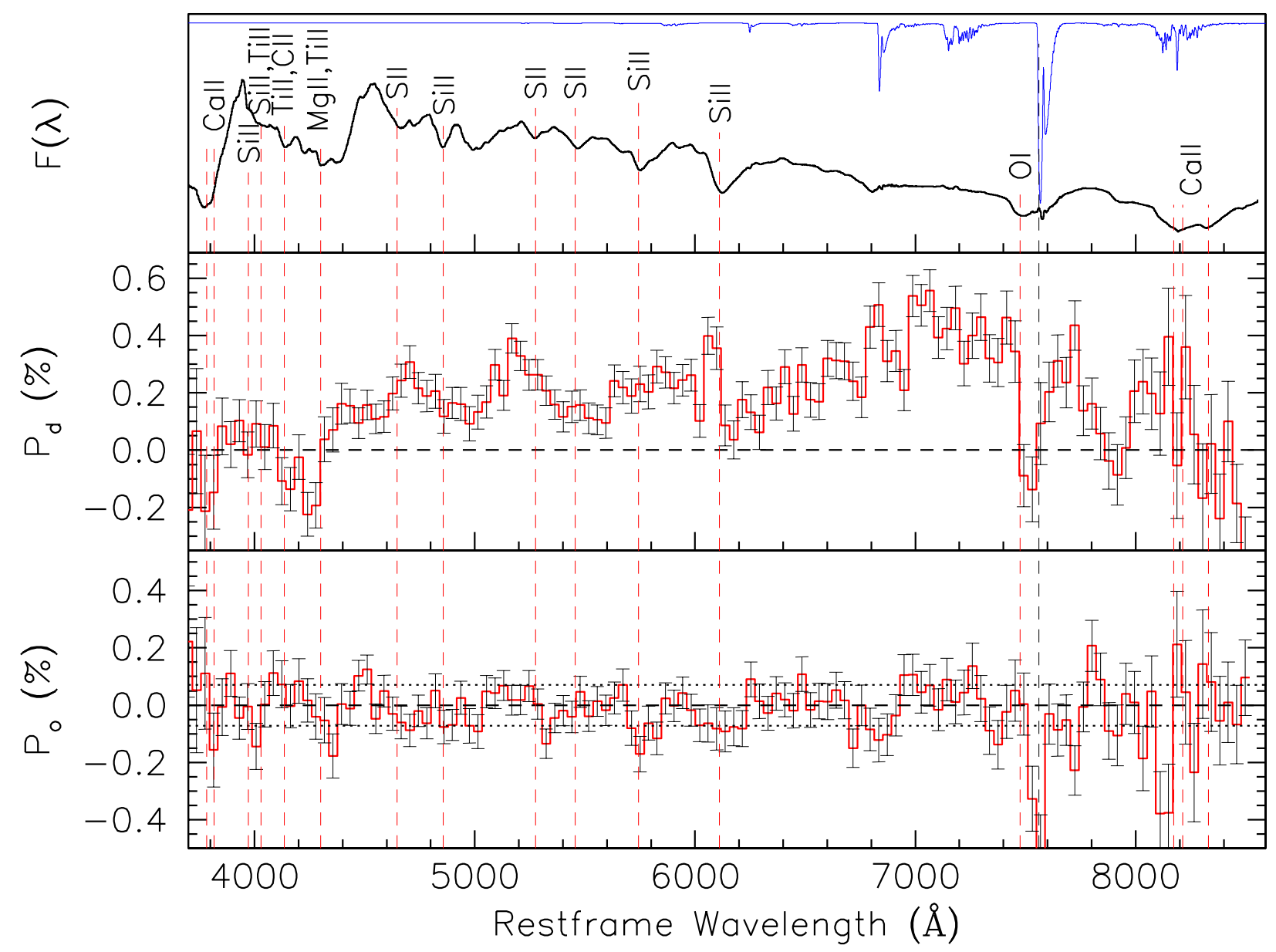

Fig. 7. Decomposition of polarization in the combined pre-maximum data set along the dominant (central panel) and orthogonal (lower panel) axis. The upper panel shows the un-binned flux spectrum and main line identifications; the thin curve traces a synthetic telluric absorption spectrum. The horizontal dotted lines in the lower panel indicate the rms deviation from null polarization.

Table 3. Best fit dominant axis parameters.

\begin{tabular}{lccc}
\hline \hline \multicolumn{4}{c}{ Least squares fit analysis } \\
\hline$\alpha$ & $-0.19 \%(0.01 \%)$ & $\beta$ & $1.10(0.13)$ \\
$\chi^{2} /$ d.o.f. & $140 / 85$ & $r_{x y}$ & 0.43 \\
$\sigma$ & $0.16 \%$ & $\theta_{\mathrm{d}}$ & $113^{\circ} .9(1.6)$ \\
\hline \multicolumn{4}{c}{ Principal components analysis } \\
\hline$\langle Q\rangle$ & $0.00(0.01)$ & $\langle U\rangle$ & $-0.20(0.01)$ \\
$b / a$ & 0.47 & $\theta_{\mathrm{d}}$ & 114.0 \\
\hline
\end{tabular}

Notes. Values in parentheses indicate rms uncertainties.

the weighted covariance matrix and extracting its eigenvectors and eigenvalues, as described in Maund et al. (2010a). The parameters retrieved through this analysis are the position angle of the dominant axis $\left(\theta_{\mathrm{d}}\right)$ and the ratio $(b / a)$ of the degrees of polarization along the dominant and perpendicular axes. The results are presented in Table 3, which includes the centroid coordinates $(\langle Q\rangle,\langle U\rangle)$.

Following Wang et al. (2003), we computed the components of the ISP-subtracted polarization along $\left(P_{\mathrm{d}}\right)$ and perpendicular $\left(P_{\mathrm{o}}\right)$ to the dominant axis, which are obtained by rotating counterclockwise the observed $Q-U$ coordinates system by the angle $\theta_{\mathrm{d}}$ that defines the dominant axis (106.1 degrees). The result is presented in Fig. 7, where we have also plotted the main line identifications, following Taubenberger et al. (2008).
The first fact that emerges from this decomposition is that the bulk of the polarization is aligned along the dominant axis. The orthogonal component $P_{\mathrm{o}}$ is statistically consistent with a null value across the whole wavelength range. One possible exception is seen at the wavelength corresponding to the absorption trough of the $\mathrm{O}_{\mathrm{I}}$ line at about $7570 \AA$. This deviation slightly exceeds the 3 -sigma level $\left(P_{\mathrm{o}} \sim-0.50 \pm 0.15 \%\right)$, and it takes place in a region which is partially affected by a strong telluric feature (see the telluric absorption spectrum in Fig. 7, upper panel), and therefore it needs to be considered with caution. Taken at face value, this would imply that oxygen is polarized along a direction orthogonal to that displayed by the underlying continuum.

The second remarkable property revealed in Fig. 7 is the relatively low polarizations corresponding to the main absorption features such as Si II $\lambda 6355$ and the NIR Ca II triplet, with respect to that typically observed in Type Ia SN a week before maximum light (Wang \& Wheeler 2008). Although there are some localized, weak peaks in $P_{\mathrm{d}}$ (some of which are statistically significant), the polarization is always below $\sim 0.5 \%$. The polarization is dominated by the continuum, which shows an increase from blue to red peaking at about $7200 \AA$. This is reminiscent of what was found for SN 1999by (Howell et al. 2001), and establishes a clear link between these two subluminous events (see Fig. 8, top and middle panels). In addition, the continuum polarization marks a clear difference with respect to core-normal events. In core-normal events, the continuum polarization in the range $6400-7000 \AA$ is always below $0.2 \%$ 


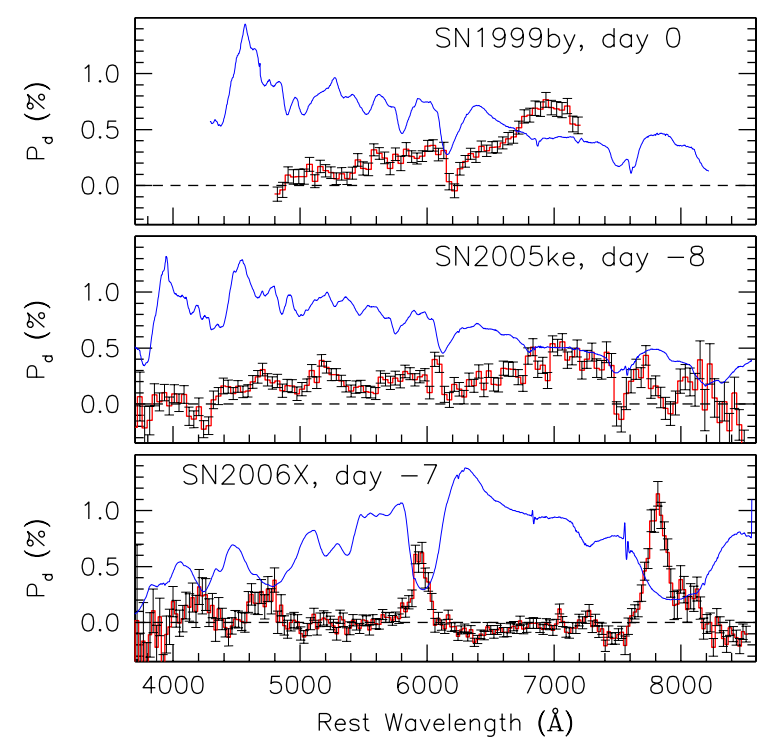

Fig. 8. Comparison between the dominant axis polarization spectra of SNe 1999by (top; Howell et al. 2001), 2005ke (middle), and 2006X (bottom; Patat et al. 2009a). The dominant component for SN 1999by was computed using $\theta_{\mathrm{d}}=80$ degrees (Howell et al. 2001). For presentation, the original 1999 by data were binned in $\sim 40 \AA$ wide bins (11 pixels). The dashed horizontal line marks the zero-polarization level. The thin (blue) lines trace the observed flux spectra. No reddening correction was applied to SN 2006X.

(see for instance SN 2006X, Patat et al. 2009a). The overall differences between the subluminous events (SN 1999by, SN 2005ke) and the core-normal SN 2006X are illustrated in Fig. 8, which presents the polarization spectra along the respective dominant axes.

The most marked feature in the dominant-axis component is the peak corresponding to the Si II $\lambda 6355$ absorption. The polarization profile, decomposed along and orthogonally to the dominant axis is shown in Fig. 9. The polarization peak $\left(P_{\mathrm{d}} \sim\right.$ $0.39 \pm 0.08 \%$ ) occurs at $-13300 \mathrm{~km} \mathrm{~s}^{-1}$, while the minimum of the absorption trough is at a lower velocity $\left(-10800 \mathrm{~km} \mathrm{~s}^{-1}\right)$, as is typical of Type Ia SNe (see for instance the well studied case of SN 2006X; Patat et al. 2009a). The polarization angle across the line is constant (within the errors), and equal to $\theta_{\mathrm{d}}$, suggesting that the line forming region and the photosphere share the same geometrical properties. The signal-to-noise ratio is insufficient to tell whether the polarization angle is constant across the NIR Ca II triplet as well.

\section{Models for SN 2005ke}

In this section, we compare the observations of SN 2005ke with possible theoretical scenarios. As in previous studies, the results have been obtained using our hydrodynamical radiation code, HYDRA. This allows us to solve the hydrodynamics including nuclear reaction networks for the explosion and radiation transport, including detailed atomic models for light curves, flux and polarization spectra. The methods are described in Höflich (1991, 1995), Howell et al. (2001), Höflich et al. (2002, 2006, 2009), Wang et al. (2006b). For calculating the polarization spectra in our Monte Carlo module, we use the given density, temperature and chemical structures including the non-LTE occupation of the atomic models. We can do so because polarization will not affect the rate equations within the accuracy of our approximations. In $\mathrm{SNe}$, most of the intrinsic polarization is due to Thomson scattering; nevertheless,

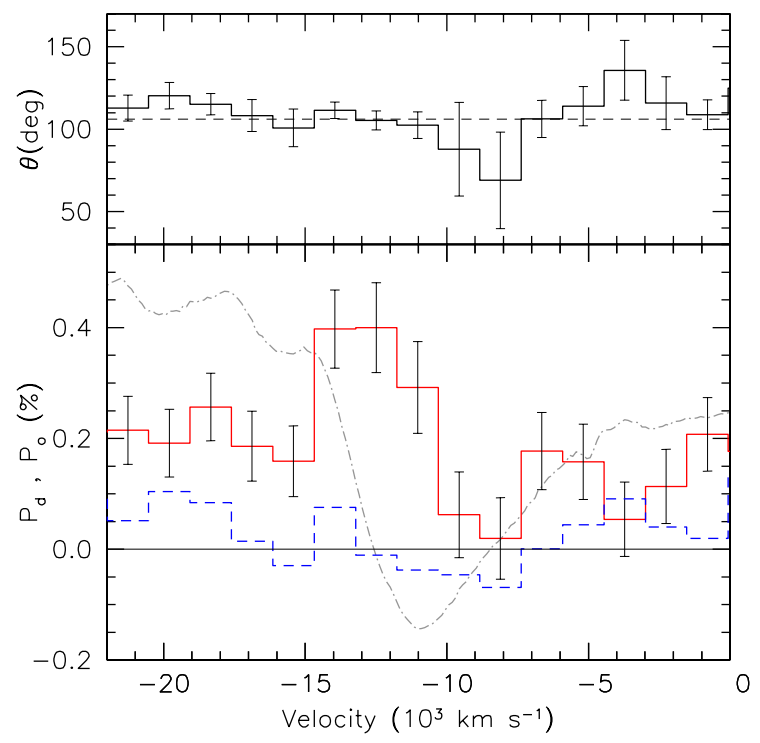

Fig. 9. Lower panel: polarization profile of $\mathrm{Si}$ II $\lambda 6355$ at the combined pre-maximum epoch. Solid and dashed lines trace $P_{\mathrm{d}}$ and $P_{\mathrm{o}}$, respectively ( 12 px bins, $\sim 35 \AA$ ). The dotted-dashed curve is the unbinned flux spectrum arbitrarily scaled. Upper panel: polarization position angle. The horizontal dashed line indicates the dominant axis angle $\theta_{\mathrm{d}}$.

our calculations take into account also Rayleigh scattering in lines, under the assumption of complete redistribution. Thus, the scattering matrix is approximated by a linear combination of Rayleigh and isotropic shift in phase (Hamilton 1947; Domke \& Hubeny 1988) with the size of polarization given by the angular momentum of the lower atomic state (Chandrasekhar 1960).

In the first part of this section, we will discuss the origin of the polarization detected in SN 2005ke. This is done in the framework of a subluminous delayed-detonation (DD) model of a Chandrasekhar mass white dwarf (WD) because many properties of SN1991bg-like events can be understood within this family of models. We note that the deflagration-detonation transition may occur both during the initial expansion phase or after a pulsation (Khokhlov et al. 1993; Höflich 1995; Höflich et al. 2002). We choose a scenario with $M_{\mathrm{WD}}=M_{\mathrm{Ch}}$ because only these models burn under sufficiently high densities to undergo electron capture as indicated by observations (Höflich et al. 2004; Motohara et al. 2006; Fesen et al. 2007; Maeda et al. 2011). We note that the evidence for electron capture has been found in core-normal and moderately subluminous SNe Ia, SN 1986G-like events, but there is a lack of observations of $91 \mathrm{bg}$-like events. Many of the arguments presented here are generic and they are also used to discuss possible alternative scenarios including mergers and edge-lit sub-Chandrasekhar mass models.

\subsection{Delayed-detonation model}

We start with a spherical model and remap that structure to aspherical grids. We can do so because the deviations from sphericity are found to be small. The remapping allows us to study various effects, such as rotation. These effects may lead to oblate, ellipsoidal structures (Eriguchi 1985) and off-center Ni distributions, as may be produced by off-center DD (Livne 1999; Gamezo et al. 2005; Höflich \& Khokhlov 2006; Röpke 2007; Kasen et al. 2009).

Within the DD scenario, the free parameters and prescriptions are: 1) the chemical structure of the exploding WD; 2) the 

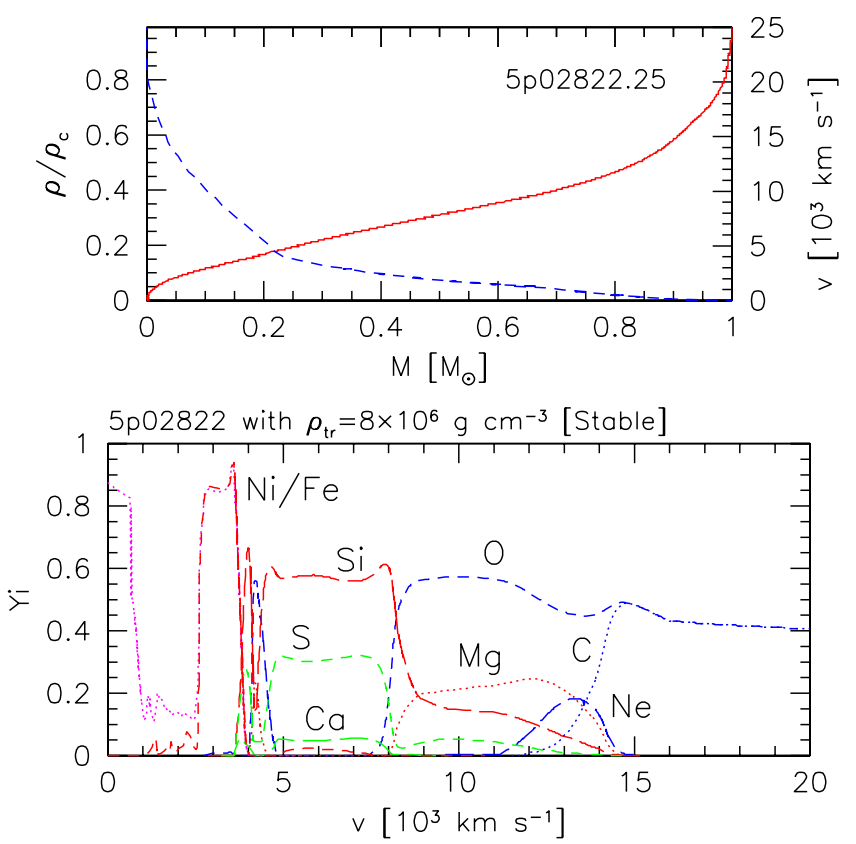

Fig. 10. Density (dotted) and velocity (solid) as a function of enclosed mass (upper panel), and abundances of stable isotopes (lower panel) as a function of the expansion velocity for DD-model $5 \mathrm{p} 0 \mathrm{z} 22.8$, with a transition density of $\rho_{\mathrm{tr}}=8 \times 10^{6} \mathrm{~g} \mathrm{~cm}^{-3}$. In addition, ${ }^{56} \mathrm{Ni}$ is given (from Höflich et al. 2002).

central density, $\rho_{\mathrm{c}}$, at the time of the explosion; 3 ) the description of the deflagration front; and 4) the density, $\rho_{\mathrm{tr}}$, at which the transition from deflagration to detonation occurs. In this work, the spherical structure of the exploding C-O WD is based on a solar metallicity model star with $5 M_{\odot}$ at the main sequence. Through accretion, this core has been grown close to the Chandrasekhar limit (see model 5p0y23z22 in Dominguez et al. 2001). At the time of the explosion of the WD, its central density $\rho_{\mathrm{c}}$ is $2.0 \times 10^{9} \mathrm{~g} \mathrm{~cm}^{-3}$ and its mass is close to $1.37 M_{\odot}$. The transition density $\rho_{\text {tr }}$ has been identified as the main factor that determines the ${ }^{56} \mathrm{Ni}$ production, and thus the brightness of a SNe Ia (Höflich 1995; Höflich et al. 1995; Iwamoto et al. 1999). The deflagration-to-detonation transition density is $\rho_{\text {tr }}=8 \times 10^{6} \mathrm{~g} \mathrm{~cm}^{-3}$. This value, which is lower than that typical of core-normal models $\left(2 \times 10^{7} \mathrm{~g} \mathrm{~cm}^{-3}\right)$, is required to produce a subluminous explosion (Höflich et al. 2002). We employ this model (5p0z22.8, Höflich et al. 2002), because it reproduces the light curves and spectra of SN 1999by from the optical to the NIR. We note that, although some $0.3 M_{\odot}$ are burned during the slow deflagration phase, only $\sim 0.09 M_{\odot}$ of ${ }^{56} \mathrm{Ni}$ are produced because of electron capture. The light curve reaches its maximum about 14.6 days after the explosion, at $M_{V} \sim-17.2$.

The final density, velocity and chemical structures are given in Fig. 10. The density and velocity profiles are very similar to core-normal SNe Ia, because the specific nuclear energy is nearly the same when burning from $\mathrm{C}-\mathrm{O}$ to $\mathrm{Si}$ - or Fe-group elements; however, more partially burned or unburned material is left in the subluminous models. Due to the larger pre-expansion during the deflagration phase, most of the star is burned to $\mathrm{Si}$-group elements rather than to ${ }^{56} \mathrm{Ni}$. This structure is consistent with those obtained by inverse spectral analysis (Mazzali et al. 1997). A further feature that distinguishes subluminous models from core-normal DD models is the extended region of unburned C-O. As discussed earlier (see also Fig. 9), the Si line profile extends in the blue up to about $16000 \mathrm{~km} \mathrm{~s}^{-1}$, consistent with the chemical structure of this model. The photosphere is at a velocity of about $10000 \mathrm{~km} \mathrm{~s}^{-1}$, i.e. well within the Si-rich zone.

\subsection{Flux spectra}

In Fig. 11, we compare spectra of SN 2005ke at 8 days before maximum with theoretical spectra at about day 9 after the explosion assuming an ellipsoidal geometry with an axis ratio $b / a=0.85$.

The model is taken directly from the time evolution of the initial explosion model without further tuning. The adopted $b / a$ axis ratio allows us to reproduce the size of the polarization in the red for inclinations between 30 and 60 degrees.

Overall, the theoretical spectra provide a good match to the observed flux spectra of SN 2005ke: the overall energy distribution, the presence and Doppler shifts of the lines are well reproduced. The spectrum is dominated by single ionized stages of Si, S, Ti and the iron group elements. Given the simplifying assumptions made, one cannot expect a perfect fit. For one, we assumed that the time evolution is identical to the spherical case, and we calculated the asymmetric profiles for a specific point in time. We had to do so because detailed atomic models for the ions are beyond our current computational means. This, however, is a good first-order approximation, again because the deviations from sphericity are small. The theoretical line strengths are slightly larger for most inclinations, which may indicate a larger than predicted population of excited levels of iron-group elements. In addition, the models have more line blocking at about $5200 \AA$, and the Ti II feature at about $4800 \AA$ is weaker than observed, as is the Ca II emission at about $3800 \AA$. From previous systematic studies of the temperature effect on spectra (Nugent et al. 1995), we estimate that the local temperature in our model is too high by about $1000 \mathrm{~K}$.

\subsection{Polarization spectra for oblate geometries}

The overall polarization agrees with the data of SN 2005ke (Fig. 11, lower panel). In the optical, $\mathrm{P}$ increases with increasing wavelength, and shows a maximum around $7000 \AA$. The largest discrepancy between the models and the data is seen at about $5200 \AA$, where the calculations predict little or no polarization, while the observations show $P \approx 0.2$ to $0.3 \%$, i.e. well outside the error bars. A missing feature is the depolarization at about $8000 \AA$ which may be attributed to shell interaction with the environment (Gerardy et al. 2004; Quimby et al. 2006), but has not been included in this model. To fit such a feature, two additional parameters would have to be added, the orientation and the mass of the circumstellar matter. Note that the seemingly high observed polarization below $3600 \AA$ is affected by very large uncertainties due to the low flux levels.

In the following, we will discuss the polarization results. In general, polarization provides information about asphericity on scales comparable to the mean free path of photons. Polarization is decreased if the radiation field becomes more isotropic, e.g. by thermalization (Höflich 1991).

The origin of line and continuum polarization in rapidly expanding atmospheres dominated by Thomson scattering can be represented by three idealized scenarios (Höflich 1991; Höflich 1995; Kasen et al. 2003). These are: 1) aspherical density distributions e.g. by rapid rotation of a single WD or binary WD mergers; 2) anisotropy in the radiation field, e.g. aspherical ${ }^{56} \mathrm{Ni}$ distributions; or 3 ) selective line blocking of the underlying "photo-disk", e.g. by aspherical chemical distributions 


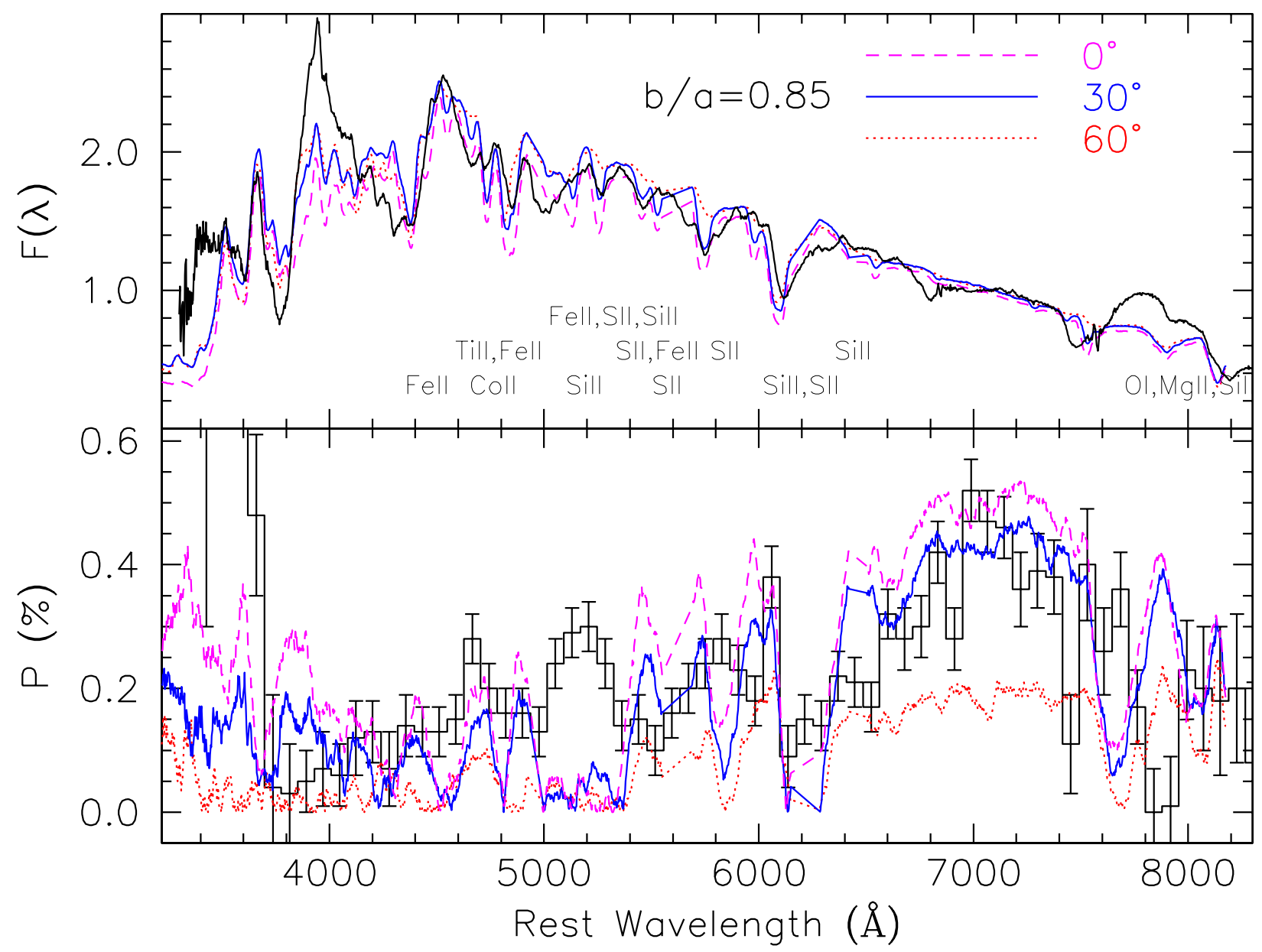

Fig. 11. Model flux (top) and polarization (bottom) spectra of the subluminous DD model $5 \mathrm{p} 0 \mathrm{z} 22.8$ at 7 days before maximum light at three inclination angles in comparison with the data of SN 2005ke ( $\sim 8$ days before maximum). We assumed an aspherical density structure with an axis ratio $b / a=0.82$. We show the observations (black, with error bars for the polarization). For presentation, the observed polarization data were binned to $78 \AA$ (30 pix).

as caused by off-center DDs. We first consider the latter mechanism. The alternative polarization mechanism will be considered in Sects. 6.1 and 6.2.

\subsubsection{Line polarization}

Selective line blocking requires a non-radial chemical gradient in the line forming region and can occur even though the Thomson scattering photosphere may be spherical. As a result, the "photo-disk" shows local polarization increasing with the distance from the center, because perpendicularly scattered photons and forward scattered photons are $100 \%$ and $0 \%$ polarized, respectively (note that, at the photosphere, not all photons are traveling radially even at the rim of a disk). For a sphere, the components of the polarization field cancel out. If the chemical distribution is asymmetric, the disk may be only partially obscured at the wavelength of the line. If seen off-axis, the line may be optically thick in one part of the disk and optically thin in another. Thus, the polarization vectors of the underlying Thomson photosphere incompletely cancel yielding a high line polarization. This effect can cause a line polarization of the order of $2 \%$ if the line-forming region is detached from the photosphere, or for strong lines in rapidly expanding photospheres of SNe.

Strong polarization in lines is produced by "asymmetric covering" of the underlying Thomson scattering-dominated photosphere by asymmetries in the abundance distribution. For strong lines, the amount of material required to selectively block the photosphere may be small. Line polarization is a transient phenomenon, dictated by the position of the receding photosphere with respect to the boundaries of chemical asymmetries (see also Höflich et al. 2006; and the discussion in Patat et al. 2009a). High line polarization levels are commonly seen in Ca II, $\mathrm{Mg}$ II, $\mathrm{S}$ II and Si II in many core-normal SNe at early times (Wang et al. 2003; Kasen et al. 2003; Wang et al. 2004, 2006b; Patat et al. 2009a; Maund et al. 2010a). In these objects, the effect is large when the photosphere is close to the chemical boundary, e.g. between the $\mathrm{Mg} / \mathrm{Ne} / \mathrm{O}$ and $\mathrm{Si} / \mathrm{S}$-rich layers. In subluminous events, the corresponding lines block the entire photosphere, because the Thomson photosphere is smaller, and the lines are formed in an extended region of $\mathrm{Si} / \mathrm{S}$ (see below).

Although the models included Thomson scattering on free electrons and Rayleigh scattering in lines, the former is larger by a factor of 10 and, for the sake of the argument, we assume that absorption in a line depolarizes the radiation field. In SN 2005ke and at -8 days, the lines are formed at about 10000 $12000 \mathrm{~km} \mathrm{~s}^{-1}$, i.e. far from chemical boundaries (see Fig. 10). Therefore, line polarization by selective line blocking is small for strong lines such as Mg II, Si II and S II. Intrinsic line polarization due to Rayleigh scattering is small for most line transitions; however, high polarization is sometimes observed in strong lines if they form in regions of asymmetric chemical distributions. The resulting asymmetric blocking of the light from the scattering 

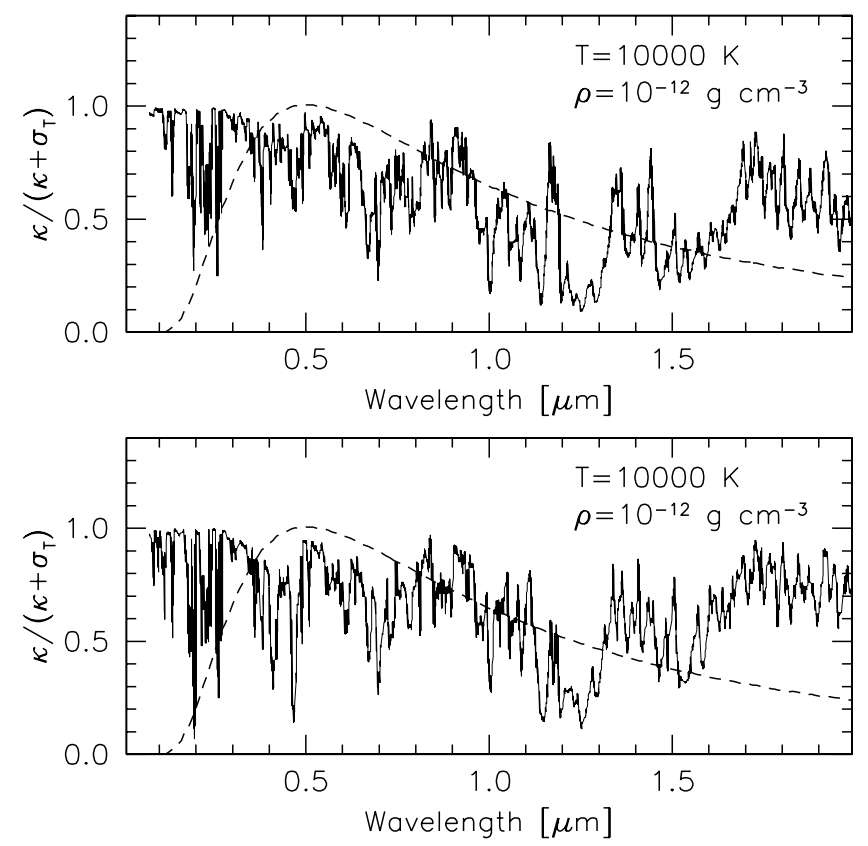

Fig. 12. Comparison of the ratio $R$ of line to total opacity for conditions typical for the photosphere of a SN Ia at the thermalization optical depth which corresponds to an optical depth of $\approx 2$ to 3 in Thomson scattering for photons in the optical and near IR. Line opacity is given at $10000 \mathrm{~K}$ and for a density of $10^{-12} \mathrm{~g} \mathrm{~cm}^{-3}$ for the Si-rich (top) and Fe-rich (bottom) composition (from Höflich 1993), corresponding to the thermalization and last scattering layers in subluminous and corenormal models, respectively. In the red (at $\approx 7000 \AA$ ) the average $R$ is higher by about $50 \%$ in the latter. The scattering optical depth at the last scattering radius is about 0.5 and 0.3 for subluminous and core-normal $\mathrm{SNe}$ Ia, respectively. As a consequence, continuum polarization will be smaller for the same degree of asphericity (see text). The dashed lines are the flux distributions for Planck functions of the corresponding effective temperature.

photosphere can result in a high net polarization. In the model for SN 2005ke, the large extension in velocity space of zones of $\mathrm{C}$ burning and incomplete $\mathrm{O}$ burning causes blocking of the entire photosphere, so strong lines forming in that region do not display polarization. Weak lines form close to the photosphere and, thus, always result in small polarization. In conclusion, the polarization spectrum is dominated by depolarization in strong lines.

\subsubsection{Continuum polarization}

In addition to the polarization for line features, it is also important to understand the polarization behavior of the continuum. In $\mathrm{SNe}$ Ia, a pseudo-continuum is formed by blending of a large number of lines which are mostly responsible for thermalization (Karp 1977). The wavelength dependence of the pseudocontinuum is one of the keys to understand the wavelength dependence of $P$. The ratio $R$ between true absorption $\kappa$ and total extinction, $\kappa+\sigma_{\text {Thomson }}$, determines the thermalization optical depth, $\tau \approx \sqrt{3 / R}$ and, more important, the ratio between polarizing, i.e. Thomson, and non-polarizing extinction at the radius of last interaction, $\tau_{\text {extinction }} \approx 1$. In Fig. $12, R$ is given for $\mathrm{Si}$ and Fe-rich mixtures for conditions typical of Type Ia SN photospheres before maximum light. Photons thermalize and are depolarized at Thomson optical depths between 0.1 to 3 and 0.1 to 1 , respectively. The ratio depends strongly on wavelength.
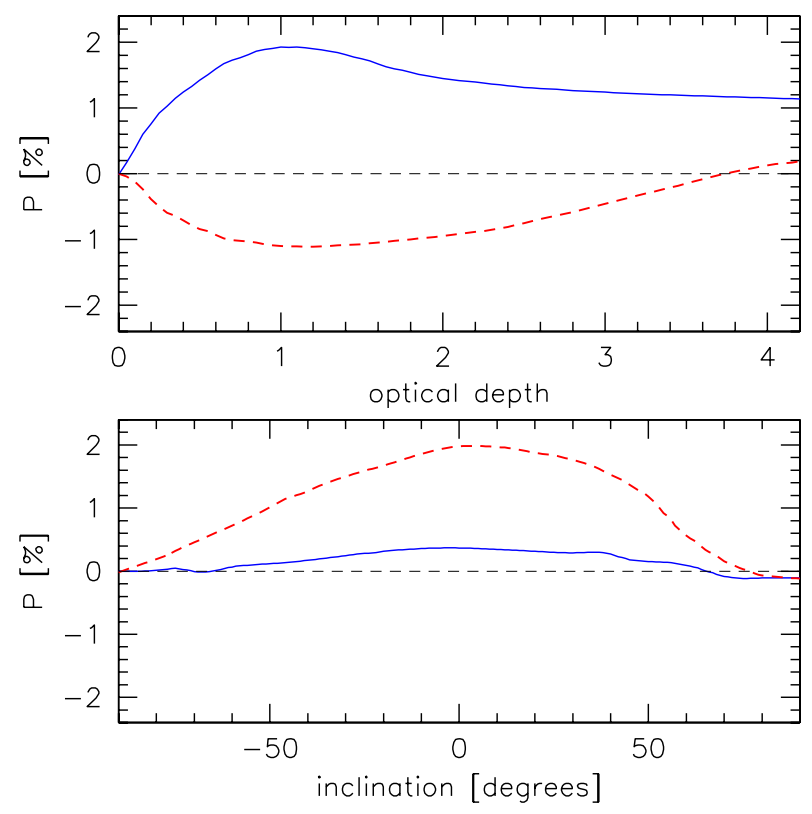

Fig. 13. Continuum polarization $P$ for aspherical configurations in scattering dominated atmospheres with an electron distribution $\propto r^{-3}$. Upper panel: maximum linear polarization as a function of the optical depth for oblate (solid blue) and prolate (dashed red) ellipsoids. Lower panel: $P$ as a function of the inclination for a source located off-center, at a distance $D=R_{\mathrm{ph}} / R$ of 3 (solid blue) and 1.5 (dotted red) (adopted from Höflich et al. 1995). Note that $D=3$ corresponds to SN 2005ke at day -8 , when the photosphere $R_{\mathrm{ph}}$ is formed at about $10000 \mathrm{~km} \mathrm{~s}^{-1}$, and assuming the maximum off-center ${ }^{56} \mathrm{Ni}$ distribution suggested by Maeda et al. (2011) for core-normal SNe Ia.

In the UV, where $R$ is close to unity, the opacities are line dominated whereas at longer wavelengths, Thomson scattering becomes more important.

A second key for the understanding of continuum polarization in scattering-dominated atmospheres is the behavior of polarization as a function of optical depth and degree of asymmetry (Höflich 1991). In Fig. 13, we show the continuum polarization $P(\tau)$ for various configurations. Let us first consider an oblate ellipsoid. If the absorptive optical depth is small, $P$ increases linearly with $\tau_{\mathrm{sc}}$ because of the increasing likelihood that a photon undergoes scattering. It reaches a maximum at $\tau \sim 1.2$ and, then, declines by about $60 \%$, as shown by the solid curve in Fig. 13 (upper panel). This decrease in polarization at larger optical depth is because, with multiple scattering, the radiation field becomes more isotropic. Note that for an oblate geometry, $P$ varies with optical depth, but does not change sign, i.e. the polarization angle is constant.

This has two important implications for our understanding of polarization spectra: 1) the continuum polarization degree is not a linear function of the opacity in the pseudo-continuum; and 2 ) the continuum polarization angle may change with wavelength even for axially symmetric configurations (i.e. $P$ may be not positive definite) for prolate structures or off-center energy sources (see Sect. 6.1). For oblate ellipsoids the wavelength dependencies of opacity and continuum polarization are related. Therefore, the wavelength dependence of $P$ seen in SN 2005ke (Fig. 11) can be understood in terms of the variation of the optical depth with wavelength of the photosphere for a Si-rich chemistry, which is relevant to subluminous $\mathrm{SNe}$ Ia (as opposed to Fe-rich, core-normal events). At short wavelengths, lines form a pseudo-continuum and dominate the opacity. As a consequence, 


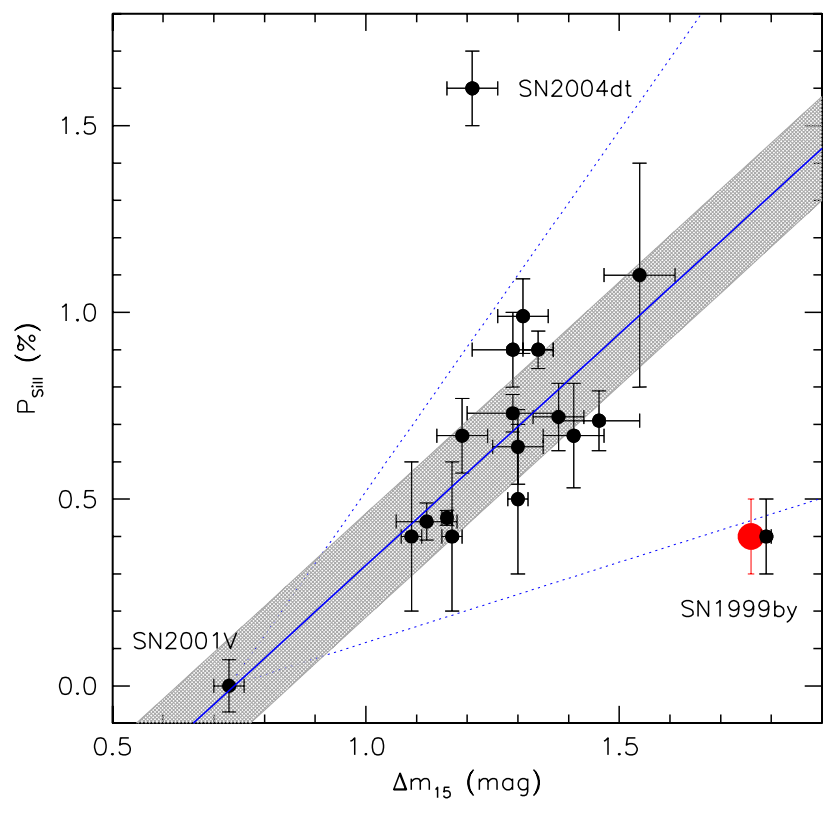

Fig. 14. Degree of polarization across the Si II $\lambda 6355$ line as a function of light curve decline rate. Data are from Wang et al. (2007) and Patat et al. (2009a). SN 2005ke is marked by the large dot close to SN 1999by. The solid line is a best fit to all data, with the exception of SN 1999by, $2004 \mathrm{dt}$, and 2005ke. The dotted lines trace the 1- $\sigma$ level of the intrinsic polarization distribution generated by the Monte-Carlo simulation discussed by Wang et al. (2007). The shaded area indicates the rms deviation of the data points from the best fit relation.

$P$ is low. Going to longer wavelengths, on average $\mathrm{e}^{3} R$ decreases to attain a minimum at about $7000 \AA(R \approx 0.5)$ and $P$ reaches its maximum.

Between 5000 and $5400 \AA$, blending depends sensitively on the temperature, as already discussed in the context of the flux spectrum (see Sect. 5.2). Therefore, the discrepancy between the model and the data seen around $5200 \AA$ (see Fig. 11, lower panel) may be explained in terms of temperature changes, because the energy differences between levels in heavy elements is comparable to the thermal energy.

In general, for oblate geometries, the polarization spectrum of SN 2005ke can be explained in terms of an intrinsically polarized continuum depolarized by lines.

\section{Discussion}

SN 2005ke is a close spectroscopic and photometric twin of SN 1999by, and both show similar polarization properties, with respect to the amplitude of the polarization $(\sim 0.6 \%)$ as well as wavelength dependency. SNe 1999by and 2005ke have very distinct polarization properties compared to core-normal events (see also Fig. 8), which place them in a fairly isolated region of the $\Delta m_{15}-P$ plot (Fig. 14). They also appear as outliers from the relation between the $\mathrm{Si}$ II velocity gradient and the associated polarization (Maund et al. 2010b).

The difference in appearance of subluminous events is caused by low photospheric temperatures in combination with

\footnotetext{
3 The curves shown in Fig. 12 are a realization at a single value of velocity, and do not include the blurring produced by the velocity gradient within the decoupling region. Although $R$ is sensitive to wavelength on small wavelength scales in Fig. 12, the photosphere forms over a range of velocities, leading to a significant velocity smearing in the effective value of $R$ in a given wavelength range.
}

layers of unburned carbon and more massive layers of the products of explosive carbon and oxygen burning.

The polarization of SN 2005ke can be understood in the framework of an oblate ellipsoidal geometry, with a degree of asymmetry of about $15 \%$. If this is typical of subluminous Type Ia events, then a spread in the apparent peak brightness by about 0.2 mag is expected, just because of the directional dependence of the luminosity.

One important question to ask is what causes the overall asymmetry in these objects, as opposed to what is seen in corenormal SNe Ia explosions like SN 2006X (Patat et al. 2009b, see also Fig. 8), and whether this can tell us something about the explosion mechanism that leads to these events.

It is tempting to attribute the asymmetry to the initial WD rotation, as first proposed by Howell et al. (2001) for SN 1999by. As a first approximation, we assume that the original degree of asymmetry is conserved during the explosion. As for mass redistribution, three dimensional calculations for SNe Ia explosions show little coupling between latitudes. When starting from a spherical WD, the resulting envelope remains spherical even if the burning is highly asymmetric (e.g. Gamezo et al. 2005). At about 7 days after the explosion, about 8 days before maximum light, the photosphere is formed in a layer enclosing $\sim 0.7 M_{\mathrm{WD}}$ (Fig. 10). Based on the work on rotating WDs (Eriguchi \& Müller 1985, 1993; Müller \& Eriguchi 1985), a rotation velocity close to the break-up velocity produces an asphericity of about $15 \%$ at layers placed at 0.7 in mass coordinates. Fast rotation may be reasonable because, within the single degenerate scenario, mass is accreted from a companion star via an accretion disk rotating at near-Keplerian velocities, although this aspect does not differentiate subluminous $\mathrm{SNe}$ from single degenerate models of core-normal events.

If the scenario in which the overall asymmetry is inherited from the WD rotation is correct, then one can reverse the argument, and speculate that a low (or null) polarization indicates a slow (or no) WD rotation. Now, for core-normal SN Ia, we have upper limits to the continuum polarization only before and around maximum light. This limit is 0.1 to $0.2 \%$ (Wang et al. 2006b; Wang \& Wheeler 2008; Patat et al. 2009a). This indicates that fast WD rotation and SN sub-luminosity may be related, marking a clear distinction with respect to core-normal explosions.

At this point one can ask: do we expect a similar degree of polarization in normal and subluminous events for the same degree of asymmetry? In core-normal Type Ia and around maximum light, the thermalization of pseudo-continuum photons in the red takes place mostly in layers of the iron group, at variance with objects like SN 2005ke, in which thermalization occurs in layers of incomplete burning. The ratio $R$ between true absorption and total extinction (see Sect. 5.3), averaged over the region $6750-7250 \AA$, is 0.7 and 0.5 in core-normal and subluminous supernovae, respectively. There are two reasons for the difference in $R$. Firstly, the thermalization layers changes from iron-rich to $\mathrm{Si} / \mathrm{S}$-rich compositions (Sect. 5.3). Secondly, for core-normal SNe Ia, the thermalization happens at layers of larger expansion velocity and, thus, smaller density leading to a further increase of $R$ (see Hoeflich et al. 1993, their Fig. 1).

Moreover, larger thermalization in core-normal SNe Ia results in an increased isotropy of the radiation field (Höflich 1991). For the same amount of asymmetry, the intrinsic polarization at about $7000 \AA$ may be smaller by up to a factor $\approx 2$ for core-normal SNe Ia (Figs. 12, 13), but not a factor 5 to 6 as indicated by early-time spectropolarimetric data. Though not 
conclusive, because of the small number of early-time observations for $\mathrm{SNe}$ Ia, there indeed may be a connection between rotation and sub-luminosity. In this respect, it is interesting to note that a relatively massive $\left(1.28 \pm 0.05 M_{\odot}\right) \mathrm{WD}$, with a rapid rotation $(P=13.2 \mathrm{~s})$ has been found in a binary system (Mereghetti et al. 2011). The companion star is a mass-loosing, hot subdwarf, from which the WD is currently accreting material.

\subsection{Prolate geometries and off-center energy sources}

Given the lack of spectropolarimetry time coverage, alternative geometries within $M_{\mathrm{Ch}}$ explosions need to be considered, namely prolate ellipsoids and off-center energy sources. These can be understood within the same context discussed in Sect. 5.3. In prolate geometries, tangential rays dominate the polarization for small optical depths causing negative $P$. With increasing optical depth, multiple scattering causes the radiation field to become more isotropic. This reduces the tangential flux and causes $P$ to change sign at greater optical depth (Fig. 13, and Höflich 1991, 1995).

In the light of these considerations, we first note that overall prolate structures can be ruled out for SN 2005ke. The observed polarization is positive at all wavelengths whereas, in the case of a prolate geometry, it should change its sign (i.e. angle) at wavelengths dominated by Thomson scattering (Fig. 13).

As for off-center energy sources (i.e. ${ }^{56} \mathrm{Ni}$ ) in Type Ia $\mathrm{SNe}$, these have been inferred from the NIR line profile at $1.65 \mu \mathrm{m}$ (Maeda et al. 2011), and from observations of the remnant of S-Andromedae, which has been attributed to the class of SN 1986G-like objects (Fesen et al 2007). The amount of "offcenterness" is less than about $2000 \mathrm{~km} \mathrm{~s}^{-1}$. The observations of remnants and NIR line asymmetries are limited to a few cases only, none of which is a SN $1991 \mathrm{bg}$-like event. For DD models, a week before maximum, the optical depth of the central region is about 50 (Höflich 1995). At -8 days, the inner layers cannot contribute to the emission because their diffusion time scales are larger than the expansion time. Therefore, it is not possible to distinguish global density asymmetries and ${ }^{56} \mathrm{Ni}$ asymmetries based on pre-maximum spectra.

Nevertheless, we regard off-center explosion an unlikely source of the polarization observed in SN 2005ke. If an offcenter explosion is causing the early continuum polarization, the polarization should increase until the photosphere has receded to the Ni layers at about 2 weeks after the explosion (i.e. around maximum light) and, then, the polarization angle should flip. Our late time spectropolarimetry of SN 2005ke does not show this effect, lending support to the conclusion that global density and not energy input asymmetries are at the origin of the continuum polarization displayed by this object.

Chemical asymmetries would cause large polarization in lines and no continuum polarization. Therefore, in the case of subluminous events, where the lines form far from chemical boundaries, the observed continuum polarization requires the density distribution to be asymmetric (see Sects. 5.3.1, 5.3.2).

\subsection{Sub-Chandrasekhar mass explosions and mergers}

It is still under debate whether core-normal and subluminous Type Ia are a single class (Höflich et al. 2002), or form separate groups (Hillebrandt et al. 2010; Sim et al. 2010; Röpke et al. 2011). Both sub-Chandrasekhar mass WDs and WD mergers have been suggested as possible mechanisms.
In sub-Chandrasekhar mass models the explosion is triggered by a detonation in the He-layers on top of a low mass WD which triggers the detonation of the $\mathrm{C}-\mathrm{O}$ core. Explosive He burning is much more energetic, and the resulting structures of all models show either a Ni layer (Höflich \& Khokhlov 1996; Nomoto et al. 1997; Höflich 1997; Woosley 1997; Woosley \& Kasen 2011) or, with some fine-tuning, strong Fe- and Ti-rich layers (Sim et al. 2010; Woosley et al. 2011). As a generic feature, this class of models show a ${ }^{56} \mathrm{Ni}$ core, surrounded by a layer enriched in $\mathrm{Si}-\mathrm{S}$, subsequent layers of $\mathrm{O}-\mathrm{Mg}-\mathrm{Ne}, \mathrm{C}-\mathrm{O}$, $\mathrm{Ni}-\mathrm{Fe}-\mathrm{Ti}$, and an outer layer of high velocity He. Woosley \& Kasen (2011) show that maximum light spectra look reasonable because the spectra are formed in $\mathrm{Si} / \mathrm{S}$ layers at a depth of about $0.1 M_{\odot}$. Successful models must, however, agree at all phases. In SN 2005ke at day -8 , the spectra are formed in the very outer layers, but we do not detect any of the chemical signatures predicted by any of the sub-Chandrasekhar mass models.

Merger of two WDs (Iben \& Tutukov 1984; Webbink 1984; Benz et al. 1990; Pakmor et al. 2010, 2011) has recently drawn attention because of perceived advantages for solving the Type Ia progenitor problem (Ruiter et al. 2009; Schaefer \& Pagnotta al. 2012). There is evidence that mergers may form a subclass among SNe Ia (Höflich \& Khokhlov 1996; Quimby et al. 2007; Pakmor et al. 2010, 2011).

Merger models can show a layered chemical structure similar to SD models of $M_{\mathrm{Ch}}$ explosions (details depend on whether or not material from the accreted WD is assumed to detonate). There are two aspects that qualitatively distinguish merger models from SD models: 1. late-time IR spectra and the remnant to S-Andromeda show flat topped profiles that seem to require central densities beyond $10^{9} \mathrm{~g} \mathrm{~cm}^{-3}$ in order to have electron capture time-scales comparable to hydrodynamical timescales. This condition is required to have a ${ }^{56} \mathrm{Ni}$ depletion in the center (Höflich et al. 2004; Maeda et al. 2011); 2. merger models tend to produce a significant amount of unburned carbon in the outer layers. Core-normal SNIa show little or no unburned carbon (Marion et al. 2006; Parrent et al. 2011; Silverman \& Filippenko 2012). These arguments argue against a merger origin for the majority of core-normal (and SN1986g-like in the case of S And) SNe Ia. For the subluminous SN 2005ke, these discriminators against merger models cannot be applied. Even explosions of single-degenerate WDs such as DD-models for SN 1999by show strong carbon lines in the NIR down to about $14000 \mathrm{~km} \mathrm{~s}^{-1}$. The existence of carbon does not sway the argument one way or the other. Moreover, we have no late-time NIR spectra for SN 2005ke or for any other subluminous SNe Ia and therefore no constraint on the central density in the progenitor WDs. Although we need a WD with a mass close to $M_{\mathrm{Ch}}$ for SNe 1991bg, 1999by and 2005ke to explain their light curves and spectral evolution (Höflich 2002), we cannot rule out an object with similar but slightly lower mass (Mazzali et al. 1997; Taubenberger et al. 2008). In the context of the current work, the relatively high degree of continuum polarization observed in the subluminous SNe 1999 by and 2005ke could imply a high degree of rotation in the progenitor and hence may be consistent with a double-degenerate merger, for which the explosion would be intrinsically asymmetric (e.g. Pakmor et al. 2011).

We may expect that mergers induce strong differential rotation and, thus, larger asymmetries well after maximum light (Eriguchi \& Müller 1985). However, hydrodynamical models for mergers evolved to the phase of free expansion are still missing. In general, we would expect large asphericity in the central region for mergers, but almost spherical cores for rotating WDs. 
In light of these considerations and the available data, we cannot rule out a merger for SN $2005 \mathrm{ke}$.

Time sequences of spectropolarimetric data and late-time NIR spectroscopy of subluminous events may provide a more definitive conclusion.

\section{Conclusions}

We presented and discussed the pre-maximum spectropolarimetry of the subluminous Type Ia event SN 2005ke. The main observational results can be summarized as follows:

1. SN 2005ke shows similar properties to the only other subluminous event for which spectropolarimetry is available (SN 1999by). They differ substantially from core-normal $\mathrm{SNe}$ Ia.

2. Along the dominant axis the two subluminous events display a significant continuum polarization $(0.6-0.7 \%$ at peak) that increases steadily toward the red, at variance with corenormal events, which show a low continuum polarization $(\$ 0.2 \%)$ that is essentially constant in the optical band.

3. The relatively strong polarization associated with absorption lines in core-normal events is absent in the two subluminous objects.

We interpreted these findings using our hydrodynamical radiation transfer modeling. The following conclusions emerged from this analysis:

1. The flux and polarization spectra of SN 2005ke are well reproduced by an oblate ellipsoidal geometry within a subluminous DD scenario.

2. The differences with respect to core-normal $\mathrm{SNe}$ Ia are caused by low photospheric temperatures in combination with layers of unburned $\mathrm{C}$ and more massive of the products of explosive $\mathrm{C}$ and $\mathrm{O}$ burning.

3. The comparatively large continuum polarization is explained in terms of a global asymmetry $(\sim 15 \%)$, which is not present in core-normal explosions

4. In the two subluminous events, the lines of intermediate mass elements form far from chemical boundaries and over a large velocity range compared to core-normal $\mathrm{SNe}$ Ia. This causes a blocking of the entire photosphere, resulting in weak line polarization.

5. The overall asphericity characterizing subluminous Type Ia may be produced either by a fast WD rotation, or by a double-degenerate merger.

Acknowledgements. This paper is based on observations made with ESO Telescopes at the Paranal Observatory under program IDs 076.D-0177(A) and 076.D-0178(A). The authors are grateful to ESO-Paranal staff for the support given during the service mode observations of SN $2005 \mathrm{ke}$. This work is partially based on NSF grants AST 04-06740,07-03902 \& 07-08855 to P.A.H., and AST-11-9801 to J.C.W., and AST-0708873 to L.W. The authors wish to thank an anonymous referee for constructive comments, which helped a lot to increase the clarity of the paper.

\section{References}

Appenzeller, I., Fricke, K., Fürtig, W., et al. 1998, The Messenger, 94, 1

Baek, M., Prasad, R. R., \& Li, W. 2005, IAU Circ., 8630

Benetti, S., Meikle, P., Stehle, M., et al. 2004, MNRAS, 348, 261

Benetti, S., Cappellaro, E., Mazzali, P. A., et al. 2005, ApJ, 623, 1011

Benz, W., Cameron, A. G. W., Press, W. H., \& Bowers, R. L. 1990, ApJ, 348, 647

Branch, D., Dang, L. C., Hall, N., et al. 2006, PASP, 118, 560

Bufano, F., Immler, S., Turatto, M., et al. 2009, ApJ, 700, 1456
Chandrasekhar, S. 1960, Radiative transfer (New York: Dover)

Chornock, R., Filippenko, A. V., Foley, R. J., Jha, S., \& Li, W. 2006, PASP, 118, 722

Contreras, C., Hamuy, M., Phillips, M. M., et al. 2010, AJ, 139, 519

Dominguez, I., Höflich, P., \& Straniero, O. 2001, Nucl. Phys. A, 688, 21

Domke, H., \& Hubeny, I. 1988, ApJ, 334, 527

Eriguchi, Y., \& Müller, E. 1985, A\&A, 147, 161

Eriguchi, Y., \& Müller, E. 1993, ApJ, 416, 666

Fesen, R. A., Höflich, P. A., Hamilton, A. J. S., et al. 2007, ApJ, 658, 396

Filippenko A. V., Richmond, M. W., Branch, D., et al. 1992, AJ, 104, 1543

Folatelli, G., Phillips, M. M., Burns, C. R., et al. 2010, AJ, 139, 120

Gamezo, V. N., Khokhlov, A. M., Oran, E. S., et al. 2003, Science, 299, 77

Gamezo, V. N., Khokhlov, A. M., \& Oran, E. S. 2005, ApJ, 623, 337

Garnavich, P., Bonanos, A. Z., Krisciunas, K., et al. 2004, ApJ, 613, 1120

Gerardy, C. L., Höflich, P. A., Fesen, R. A., et al. 2004, ApJ, 607, 391

Hamilton, D. R. 1947, ApJ, 106, 457

Heiles, C. 1999, AJ, 119, 923

Hillebrandt, W., \& Niemeyer, J. C. 2000, ARA\&A, 38, 191

Hillebrandt, W., \& Röpke, F. K. 2010, New Astron. Rev., 54, 201

Höflich, P. 1991, A\&A, 246, 481

Höflich, P. 1995, ApJ, 440, 921

Höflich, P. 1997, in Thermonuclear Supernovae, eds. Ruiz-Lapuente et al., NATO ASI Series C (Dordrecht: Kluwer), 486, 705

Höflich, P. 2009, AIPC Ser., 1171, 161

Höflich, P., \& Khokhlov, A. 1996, ApJ, 457, 500

Höflich, P., Müller, E., \& Khokhlov, A. 1993, A\&A, 268, 570

Höflich, P., Khokhlov, A. M., \& Wheeler, J. C. 1995, ApJ, 444, 831

Höflich, P., Gerardy, C. L., Fesen, R. A., \& Sakai, S. 2002, ApJ, 568, 791

Höflich, P., Gerardy, C. L., \& Nomoto, K. 2004, ApJ, 617, 1258

Höflich, P., Gerardy, C. L., Marion, G. H., \& Quimby, R. 2006, New Astron. Rev., 50, 470

Howell, D. A., Höflich, P., Wang, L., \& Wheeler, J. C. 2001, ApJ, 556, 302

Iben, I., \& Tutukov, A. V. 1984, ApJS, 54, 335

Immler, S. I., Brown, P. J., Milne, P., et al. 2006, ApJ, 648, L119

Iwamoto, K., Brachwitz, F., \& Nomoto, K. 1999, ApJS, 125, 439

Jehin, E., O'Brien, K., \& Szeifert, T. 2005, FORS1+2 User's Manual, VLTMAN-ESO-13100-1543, Issue 78

Karp, A. H., Lasher, G., Chan, K. L., \& Salpeter, E. E. 1977, ApJ, 214, 161

Kasen, D., Nugent, P., Wang, L., et al. 2003, ApJ, 593, 788

Kasen, D., Nugent, P., Thomas, R. C., \& Wang, L. 2004, ApJ, 610, 876

Kasen, D., Röpke, F. K., \& Woosley, S. E. 2009, Nature, 460, 869

Khokhlov, A. 1995, ApJ, 449, 695

Khokhlov, A. 2001, ApJ, submitted [arXiv:astro-ph/0008463]

Khokhlov, A., Müller, E., \& Höflich, P. 1993, A\&A, 270, 223

Koribalski, B. S., Staveley-Smith, L., Kilborn, V. A., et al. 2004, AJ, 128, 16

Leibundgut, B., Kirshner, R. K., Phillips, M. M., et al. 1993, AJ, 105, 301

Leonard, D. C., \& Filippenko, A. V. 2001, PASP, 113, 920

Leonard, D. C., Filippenko, A. V., Barth, A. J., \& Matheson, T. 2000, ApJ, 536, 239

Leonard, D. C., Filippenko, A. V., Chornock, R., \& Li, W. 2002, ApJ, 124, 2506

Li, W., Leaman, J., Chornock, R., et al. 2011, MNRAS, 412, 1441

Livne, E. 1999, ApJ, 527, L97

Maeda, K., Leloudas, G., Taubenberger, S., et al. 2011, MNRAS, 413, 3075

Maguire, K., Sullivan, M., Thomas, R. C., et al. 2011, MNRAS, 418, 747

Marion, G. H., Höflich, P., Wheeler, J. C., et al. 2006, ApJ, 645, 1392

Matheson D. S., \& Ford, V. L. 1970, MNRAS, 74, 139M

Matheson, T., Kirshner, R. P., Challis, P., et al. 2008, AJ, 135, 1598

Maund, J. R., Wheeler, J. C., Patat, F., et al. 2007a, MNRAS, 381, 201

Maund, J. R., Wheeler, J. C., Patat, F., et al. 2007b, ApJ, 671, 1944

Maund, J. R., Wheeler, J. C., Wang, L., et al. 2010a, ApJ, 722, 1162

Maund, J. R., Höflich, P., Patat, F., et al. 2010b, ApJ, 725, L167

Mazzali, P. A., Chugai, N., Turatto, M., et al. 1997, MNRAS, 284, 151

Mazzali, P. A., Benetti, S., Stehle, M., et al. 2005a, MNRAS, 357, 200

Mazzali, P. A., Benetti, S., Altavilla, G., et al. 2005b, ApJ, 623, L37

Mereghetti, S., La Palombara, N., Tiengo, A., et al. 2011, ApJ, 737, 51

Modjaz M., Li, W., Filippenko, A. V., et al. 2001, PASP, 113, 308

Motohara, K., Maeda, K., Gerardy, C. L., et al. 2006, ApJ, 652, L101

Müller, E., \& Eriguchi, Y. 1985, A\&A, 152, 325

Nomoto, K., et al. 1997, in Thermonuclear Supernovae, eds. Ruiz-Lapuente et al., NATO ASI Series C (Dordrecht: Kluwer Academic Publishers), 486, 349

Nugent, P., Phillips, M., Baron, E., Branch, D., \& Hauschildt, P. 1995, ApJ, 455, L147

Parrent, J. T., Thomas, R. C., \& Fesen, R. A. 2011, ApJ, 732, 30

Patat, F., \& Romaniello, M. 2006, PASP, 118, 146

Patat, F., Benetti, S., Cappellaro, E., et al. 1996, MNRAS, 278, 111

Patat, F., Baade, D., Taubenberger, S., \& Wheeler, J. C. 2005, IAU Circ., 8631

Patat, F., Baade, D., Höflich, P., et al. 2009a, A\&A, 508, 229 
Patat, F., Maund, J. R., Benetti, S., et al. 2009b, A\&A, 510, 108 Pakmor, R., Kromer, M., Röpke, F. K., et al. 2010, Nature, 463, 61 Pakmor, R., Hachinger, S., Röpke, F. K., \& Hillebrandt, W. 2011, A\&A, 528, A117

Quimby, R., Höflich, P., \& Wheeler, J. C. 1997, 666, 1083

Quimby, R., Höflich, P., Kannappan, S. J., et al. 2006, ApJ, 636, 400

Röpke, F. K. 2007, ApJ, 668, 1103

Röpke, F. K., Seitenzahl, I. R., Benitez, S., et al. 2011, Prog. Par. Nucl. Phys., 66,309

Ruiter, A. J., Belcynski, K., \& Fryer, C. 2009, ApJ, 699, 2026

Scarrot, S. M., Ward-Thompson, D., \& Warren-Smith, R. F. 1987, MNRAS, 224, 299

Schaefer, B. E., \& Pagnotta, A. 2012, Nature, 481, 164

Schlegel, D. J., Finkbeiner, D. P., \& Davis, M. 1998, ApJ, 500, 525

Serkowski, K., Matheson, D. S., \& Ford, V. L. 1975, ApJ, 196, 261

Silverman, J. M., \& Filippenko, A. V. 2012, MNRAS, submitted [arXiv: 1202.3788]

Sim, S. A., Röpke, F. K., Hillebrandt, W., et al. 2010, ApJ, 714, L52

Stritzinger, M., Leibundgut, B., Walch, S., \& Contardo, G. 2006, A\&A, 450, 241

Taubenberger, S., Hachinger, S., Pignata, G., et al. 2008, MNRAS, 385, 75
Tully, R. B. 1988, Catalog of Nearby Galaxies (Cambridge, UK: Cambridge University Press)

Turatto, M., Benetti, S., Cappellaro, E., et al. 1996, MNRAS, 283, 1

Vinko, J., Kiss, L. L., Csak, B., et al. 2001, AJ, 121, 3127

Wang, L. 2005, ApJ, 635, L33

Wang, L., \& Wheeler, J. C. 1996, ApJ, 462, L27

Wang, L., \& Wheeler, J. C. 2008, Ann. Rev. Astr. Ap., 46, 433

Wang, L., Wheeler, J. C., Li, Z., \& Clocchiatti, A. 1996, ApJ, 467, 435

Wang, L., Wheeler, J. C., \& Höflich, P. 1997, ApJ, 476, L27

Wang, L., Howell, D. A., Höflich, P., \& Wheeler, J. C. 2001, ApJ, 550, 1030

Wang, L., Baade, D., Höflich, P., et al. 2003, ApJ, 591, 1110

Wang, L., Baade, D., Höflich, P., et al. 2004, ApJ, 604, L53

Wang, L., Baade, D., Patat, F., \& Wheeler, J. C. 2006a, CBET, 396

Wang, L., Baade, D., Höflich, P., et al. 2006b, ApJ, 653, 490

Wang, L., Baade, D., \& Patat, F. 2007, Science, 315, 212

Webbink, R. F. 1984, ApJ, 277, 355

Whittet, D. C. B., Martin, P. G., Hough, J. H., et al. 1992, ApJ, 386, 562

Wood-Vasey, W. M., Friedman, A. S., Bloom, J. S., et al. 2008, ApJ, 689, 377

Woosley, S. 1997, in Thermonuclear Supernovae, eds. Ruiz-Lapuente et al.,

NATO ASI Series C (Dordrecht: Kluwer Academic Publishers), 486, 313

Woosley, S. E., \& Kasen, D. 2011, ApJ, 734, 38 